\title{
State Feedback Fuzzy Adaptive Control for Active Shimmy Damping
}

\author{
Gaétan Pouly*, Thai-Hoang Huynh, Jean-Philippe Lauffenburger, Michel Basset
}

In the context of aircraft, shimmy is an oscillatory phenomenon of the landing gear mainly due to the tire and the landing gear structural dynamics. This phenomenon which can result in severe damage to the landing gear must be damped. This paper presents a new nose landing gear model including an actuator model and a simple tire/road interface description approximating the Pacejka model to allow the active damping of the shimmy phenomenon. Two state feedback control solutions, based on indirect and direct fuzzy adaptive theories, are also presented and compared with a more classic Proportional Integral Derivative (PID) solution. Results corresponding to different test scenarios and robustness analysis show that the proposed controllers are able to efficiently damp the shimmy phenomenon, unlike the PID controller.

Keywords: Adaptive control, fuzzy estimation, feedback linearization, nonlinear systems, active shimmy damping.

\section{Introduction}

The shimmy phenomenon is the self-excited oscillation of a wheel about its vertical steering axis, which may occur in many physical rolling systems such as aircraft nose wheels, automobiles, motorcycles. This phenomenon is particularly well-known for its non-linear chaotic dynamics [25] and could for instance be adequately represented by a dimensionless formulation. Shimmy is a violent,

*Correspondence to: G. Pouly, E-mail: gaetan.pouly@gmail.com possibly dangerous vibration that can cause system malfunctions or even damages. In order to increase the understanding of this phenomenon on aircraft Nose Landing Gears (NLG), researchers have designed different shimmy models. These white boxes models are mainly based on different ways of defining the elasticity of the tires, which plays a fundamental role in the dynamics involved [22], [24] and [26]. The system under study is nonlinear exclusively because of the tire characteristics. So the tire plays a very important role in the system's behavior. In the literature, several models of the tire/ground interface could be found ([20], [17], [19]). There are essentially two ways to describe the elastic lateral dynamics of the tires: the first one is a point contact approach (Moreland's tire model, for instance) assuming that the ground/tire interaction occurs via a single point; the second one is based on the elastic string theory defining a physical tire model by an elastic string. These tire descriptions, associated with a NLG model, help to analyze the stability and the shimmy oscillation characteristics and to synthesize passive and/or active shimmy dampers. Studies have shown similar results for these two approaches [22].

A classic solution to avoid shimmy is to increase the stiffness of the NLG by changing its material for instance, and to increase the damping constant by using additional passive dampers, for instance [1]. One of the main drawbacks of such shimmy passive damping solutions is that under changing load conditions or ground-tire interfaces, they may not be efficient because of the fixed structural

Received 8 October 2009; Accepted 13 September 2010 Recommended by N. K. Kazantzis, D. Normand-Cyrot 
parameters of the NLG. Then, considering the limitations of passive damping, active solutions may be investigated, in order to take account of the changing conditions. Due to the improvement of the embedded systems (microprocessor efficiency, field bus integration,....) such active control solutions are conceivable.

In the general context of active damping of oscillatory systems, different control approaches have been used, depending on the models of the oscillatory phenomena. For an oscillatory system described by a linear second order model, simple methods such as velocity feedback control, PID control, or linear filter were first adopted to adjust the damped ratio of the system [9] and [8]. Then, modern control theories such as optimal control, adaptive control, robust control, fuzzy control or neural networks were used to design damping controllers for more complex oscillatory systems [5], [6] and [11].

Focusing on the active damping of the shimmy phenomena, different solutions have been proposed recently. Basically, active shimmy damping solutions rely on the use of sensors measuring the NLG behaviors, and a feedback control algorithm to calculate the damping moments generated by an anti-shimmy actuator. From the control theory point of view, active damping solutions would increase the system performance as the damping moments are generated, based on feedback measurement signals corresponding to real operating conditions. Even if the active control of the landing gear started in the 70s, only few studies ([2], [28], [7]) have been carried out on active shimmy damping and until now, no aircraft has been equipped with such a device. In [2], a feedback signal proportional to the vertical angular velocity of the NLG wheel is used to control the pressure of a hydraulic actuator. The switching control method is applied to stabilize the NLG dynamics in [28]. In [7], the authors suggested a control algorithm for a simple nonlinear model of the NLG based on the feedback linearization law. One drawback of the above solutions is that the control designs are based on a nominal shimmy model. However, it is difficult to consider that the behavior of the NLG stays close to its nominal behavior. In fact, the system is time-varying, the vertical load affects the response of the system, the tire dynamic is time-dependent and varies according to the ground/wheel interface. Therefore, the control performance may change following the behavior variations. Recently, there has been a strong development in the adaptive control of nonlinear systems using neural networks and fuzzy systems, not only in theory, but also in applications. Different authors have described fuzzy adaptive controllers based on backstepping ([27], [3] and [4]). These solutions help to reduce the adapted on line parameters and thus reduce the computation time of the algorithm. [16] proposed a hybrid solution using $H_{\infty}$ control to suppress the influence of external disturbances. However, these solutions need a particular class of nonlinear system (expressed in a strict feedback form), which is not adapted to shimmy control. Finally, [23] or [13] suggested fuzzy adaptive algorithms with no restriction on the system structure. So, the ability of overcoming the uncertainties and time-varying dynamics of such an algorithm is a solution to the active shimmy damping problem. Two approaches may be distinguished in the design of a state feedback fuzzy adaptive controller: direct or indirect approaches. For the direct solution, the fuzzy system is adjusted directly to ensure the control objectives, while the indirect adaptive approach uses two fuzzy systems to estimate the plant dynamics and then calculate the control law.

One of the contribution of this paper is the extension of the well known nonlinear shimmy model described by Somieski. On the one hand, an electrical actuator model is added in order to develop active control solutions. On the other hand, the initial tire model used by Somieski (based on a piecewise linear representation of the lateral force) is replaced by a nonlinear description of the lateral force function of the slip angle. The second contribution concerns a control method based on feedback linearization. The applicability of feedback linearization is limited due to the necessity of a detailed knowledge of the system dynamics in order to synthesize an accurate nonlinear controller. To cope with this drawback, adaptive nonlinear controllers are proposed. The aim of this study is to design state feedback control laws to drive the system output $y$, here the wheel angle around the vertical axis $\left(\psi_{w}\right)$, tracking a given reference output $y_{m}$ here fixed to $0^{\circ}$. In the following, it is assumed that all the states of the system are measurable and available for feedback. This assumption is a limitation for the practical and real time implementation of the solution since, in practice, a number of physical data are difficult to obtain. However, this paper presents a first contribution concerning active shimmy damping using an electromechanical actuator. In this context, this study helps to evaluate if active shimmy damping is possible considering the most favorable conditions before the generalization to more realistic conditions (with less data available, noisy measurements, etc ...) and also to define the global specifications of the electromechanical actuator (required control torque, bandwidth, energetic aspects,...). Moreover, it helps to obtain a reference (showing the best performance which could be obtained in an ideal case), which can be used to evaluate future developments.

Direct and indirect methods have been developed here and validated through different simulation scenarios (pulse disturbance applied on the wheel corresponding to a tire damage, rough runway, etc...) with varying speed conditions. To highlight the efficiency of the controllers, a comparison has also been performed with a simplePID solution. The latter has been tested, considering the same NLG 
nonlinear model and has revealed the need for more evolved control structures. Finally, the validity of the control solutions and the sensitivity of the model with respect to varying parameters are analyzed thanks to a robustness study.

The present study is carried out in the context of the European DRESS project ("Distributed and Redundant Electromechanical nose gear Steering System"). Its goal is to develop a distributed and redundant electrical steering system for an aircraft NLG which will provide improved competitiveness and aircraft safety. One of the objectives of the DRESS project, in direct link with this paper, is to analyze the impact of the new electrical steering system on the shimmy phenomenon and to study different methods for shimmy damping.

The paper is organized as follows: the nonlinear NLG model is described in Section 3. The direct and indirect fuzzy adaptive controllers are presented in Section 4. The simulation results of the two active shimmy damping solutions and the PID solution are given in Section 5 and a discussion concerning these results is proposed. Finally, Section 6 concludes this paper.

All the notations used in this paper are summarized in Section 2.

\section{Glossary}

$M_{1}$ : torsional moment provided by the torque link [N.m], $k_{s}$ : stiffness constant of the NLG [N.m. $\left.\mathrm{rad}^{-1}\right]$,

$\psi_{a}$ : rotation angle of the turning tube $[\mathrm{rad}]$,

$\psi_{w}$ : rotation angle of the wheel $[\mathrm{rad}]$,

$M_{2}$ : damping moment from viscous friction in the bearing of the oil-pneumatic shock absorber $[N . m]$,

$k_{d}$ : damping constant of the NLG $\left[N . \mathrm{m.rad}^{-1} . s\right]$,

$\dot{\psi}_{a}$ : rotation speed of the turning tube $\left[\mathrm{rad} . \mathrm{s}^{-1}\right]$,

$\dot{\psi}_{w}$ : rotation speed of the wheel $\left[\mathrm{rad} . \mathrm{s}^{-1}\right]$,

$J_{a}:$ inertia of the actuator $\left[\mathrm{kg} \cdot \mathrm{m}^{2}\right]$,

$J_{z}$ : inertia of the NLG $\left[\mathrm{kg} \cdot \mathrm{m}^{2}\right]$,

$B_{a}$ : viscous friction constant of the actuator $\left[N . m . \mathrm{rad}^{-1} . s\right]$,

$M_{3}$ : tire moment caused by the lateral tire deformations due to side slip [N.m],

$M_{z}$ : self-aligning moment $[N . m]$,

$F_{y}$ : lateral force $[N]$,

$e$ : caster length $[m]$,

$\alpha$ : slip angle $[\mathrm{rad}]$,

$F_{z}$ : vertical load $[N]$,

$v:$ A/C speed $\left[m . s^{-1}\right]$,

$M_{4}$ : tire damping moment related to the yaw rate $[N . m]$,

$y_{l}$ : lateral displacement of the wheel $[m]$,

$a$ : half of the contact length $[m]$,

$c_{F_{\alpha}}$ : cornering stiffness normed by the vertical load $\left[\mathrm{rad}^{-1}\right]$,

$c_{M_{\alpha}}$ : self aligning stiffness normed by the vertical load $\left[\mathrm{m} . r a d^{-1}\right]$, $\kappa:$ constant of tread width tire moment $\left[N . \mathrm{m}^{2} \cdot \mathrm{rad}-1\right]$

$\delta$ : limiting slip angle for lateral force $[\mathrm{rad}]$,

$\alpha_{g}$ : limiting slip angle for aligning moment $[\mathrm{rad}]$,

$\sigma:$ relaxation length of tire deflection $[m]$.

\section{Shimmy Modeling for Active Damping}

This paper proposes an extension of the well known nonlinear shimmy model presented in [22], for the design of active damping controllers. This extension is based on the integration of an actuator model and an improvement of the tire/road interface. The system considered consists of the mechanical dynamics of an electromechanical actuator, the torsional dynamics of the NLG, and the forces and moments describing the tire's elasticity. For the latter, the piecewise linear representation of the lateral effort versus the slip angle has been replaced by a nonlinear formulation. The diagram of this model which considers the actuator, the turning tube, the sliding tube and finally the tire/road interface is illustrated in Fig. 1 and is detailed in the next section.

\subsection{Nonlinear Mathematical Formulation}

The input of the model is the control torque $u$ which must be provided by an electromechanical actuator and the output of the model is the yaw angle of the wheel $\psi_{w}$ about its vertical rotating axis. The link between the actuator and the turning tube is assumed to be rigid, the angle of the actuator output $\psi_{a}$ is equal to the angle of the turning tube. Applying Newton's second law to the rotating movements of the actuator and the NLG leads to the following equations:

$$
\left\{\begin{array}{l}
J_{a} \ddot{\psi}_{a}=u-B_{a} \dot{\psi}_{a}-M_{1}-M_{2} \\
J_{z} \ddot{\psi}_{w}=M_{1}+M_{2}+M_{3}+M_{4}
\end{array}\right.
$$

where $M_{1}=k_{s}\left(\psi_{a}-\psi_{w}\right)$ is the torsional moment provided by the torque link, $M_{2}=k_{d}\left(\dot{\psi}_{a}-\dot{\psi}_{w}\right)$ is the damping moment from viscous friction in the bearings of the oilpneumatic shock absorber, $M_{3}$ is the tire moment caused by the lateral tire deformations due to side slip and $M_{4}$ is the tire damping moment related to the yaw rate, $J_{a}$ is the inertia of the actuator, $J_{z}$ is the inertia of the NLG and $B_{a}$ is the viscous friction constant of the actuator. Considering the tire dynamics, the following equations summarize the nonlinear characteristics of the tire, which are discussed in detail in [22]:

$$
\begin{aligned}
M_{3} & =M_{z}-e F_{y} \\
F_{y} & = \begin{cases}c_{F \alpha} \alpha F_{z} & \text { for }|\alpha| \leq \delta \\
c_{F \alpha} \delta F_{z} \operatorname{sign}(\alpha) & \text { for }|\alpha|>\delta\end{cases}
\end{aligned}
$$




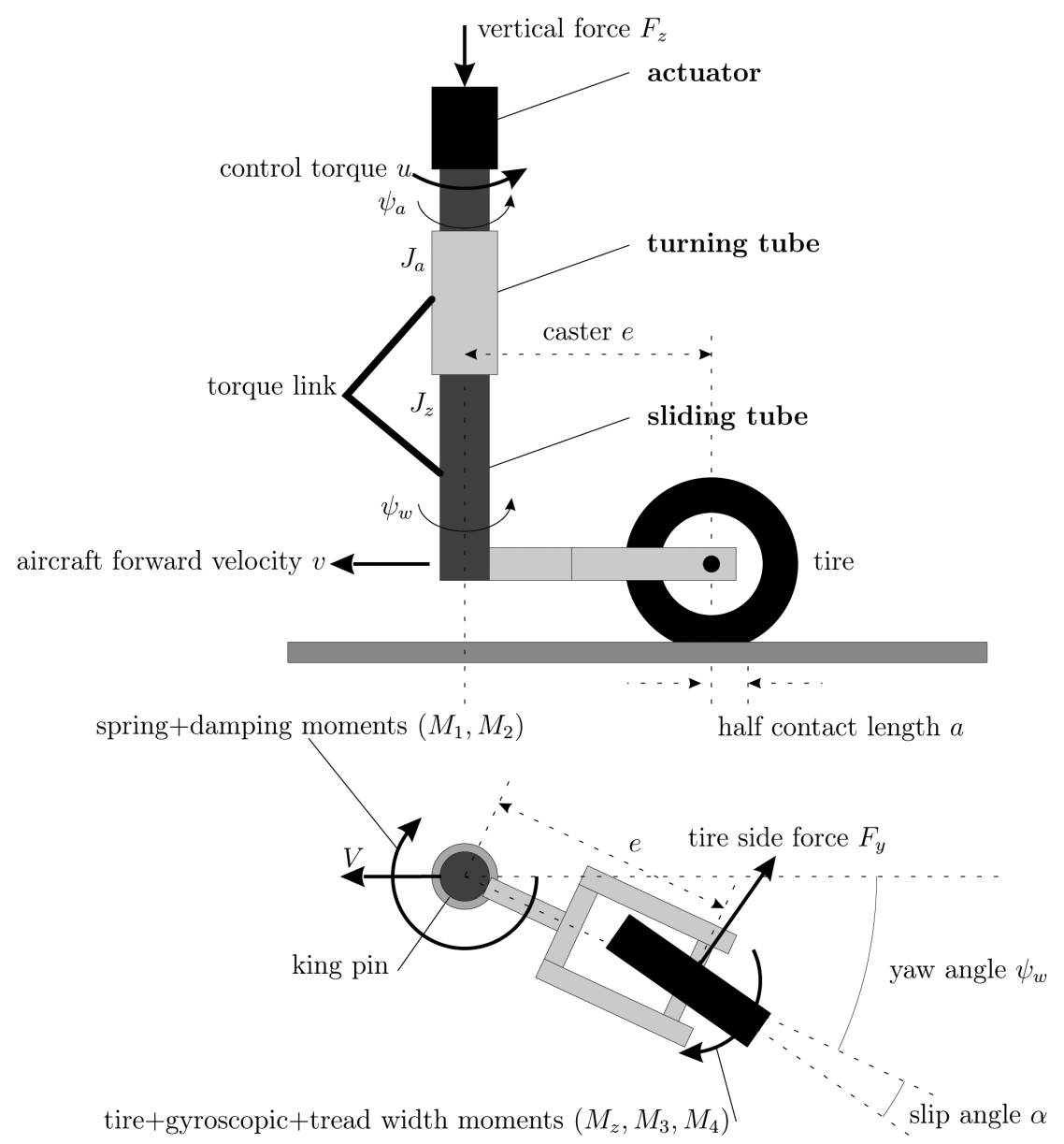

Fig. 1. Nose Landing Gear model.

$$
\begin{gathered}
M_{z}= \begin{cases}c_{M \alpha} F_{z} \frac{\alpha_{g}}{180} \sin \left(\frac{180}{\alpha_{g}} \alpha\right) & \text { for }|\alpha| \leq \alpha_{g} \\
0 & \text { for }|\alpha|>\alpha_{g}\end{cases} \\
M_{4}=\frac{\kappa}{v} \dot{\psi} \\
\dot{y}_{l}+\frac{v}{\sigma} y_{l}=v \psi_{w}+(e-a) \dot{\psi}_{w} \\
\alpha \approx \arctan \alpha=\frac{y_{l}}{\sigma}
\end{gathered}
$$

where $M_{z}$ and $F_{y}$ are respectively the self aligning torque and the lateral side force of the tire, $F_{z}$ is the vertical load applied on the NLG, $v$ is the aircraft forward velocity, $y_{l}$ is the lateral displacement of the tire footprint. This lateral displacement is obtained using the elastic string theory defining the tire lateral deformation as a stretched elastic string. Finally, $\alpha$ is the slip angle of the tire, $e$ is the caster length, $a$ is half of the contact length, $c_{F_{\alpha}}$ is the cornering stiffness normed by the vertical load, $c_{M_{\alpha}}$ is the aligning stiffness normed by the vertical load, $\kappa$ is the tread width

tire moment constant, $\delta$ is the limit slip angle for lateral forces (more details available in [22]).

It is important to note that there are two nonlinearities in the model, both related to the elasticity of the tires. These nonlinearities may cause limit cycles and instabilities in the system. Therefore, the NLG is rather difficult to control.

The precise description of the shimmy phenomena requires great accuracy in the representation of the elastic properties of the tires. In fact, shimmy is due to dynamic reaction forces between the tires and the ground. The physical elements to be considered are mainly the self aligning torque $M_{z}$ and the lateral force $F_{y}$ applied on the tire with caster length $e$ as the lever arm (see (2)). $M_{z}$ and $F_{y}$ directly depend on the load (vertical force) $F_{z}$ applied on the tire and the slip angle $\alpha$. Practically, the general shapes of the curves are shown in Fig. 2 [19].

If, for the self aligning torque $M_{z}$, the model proposed by Somieski using a half period of a sine approximates well the tire behavior in the considered working range, the saturation function with linear pieces used for the modeling 


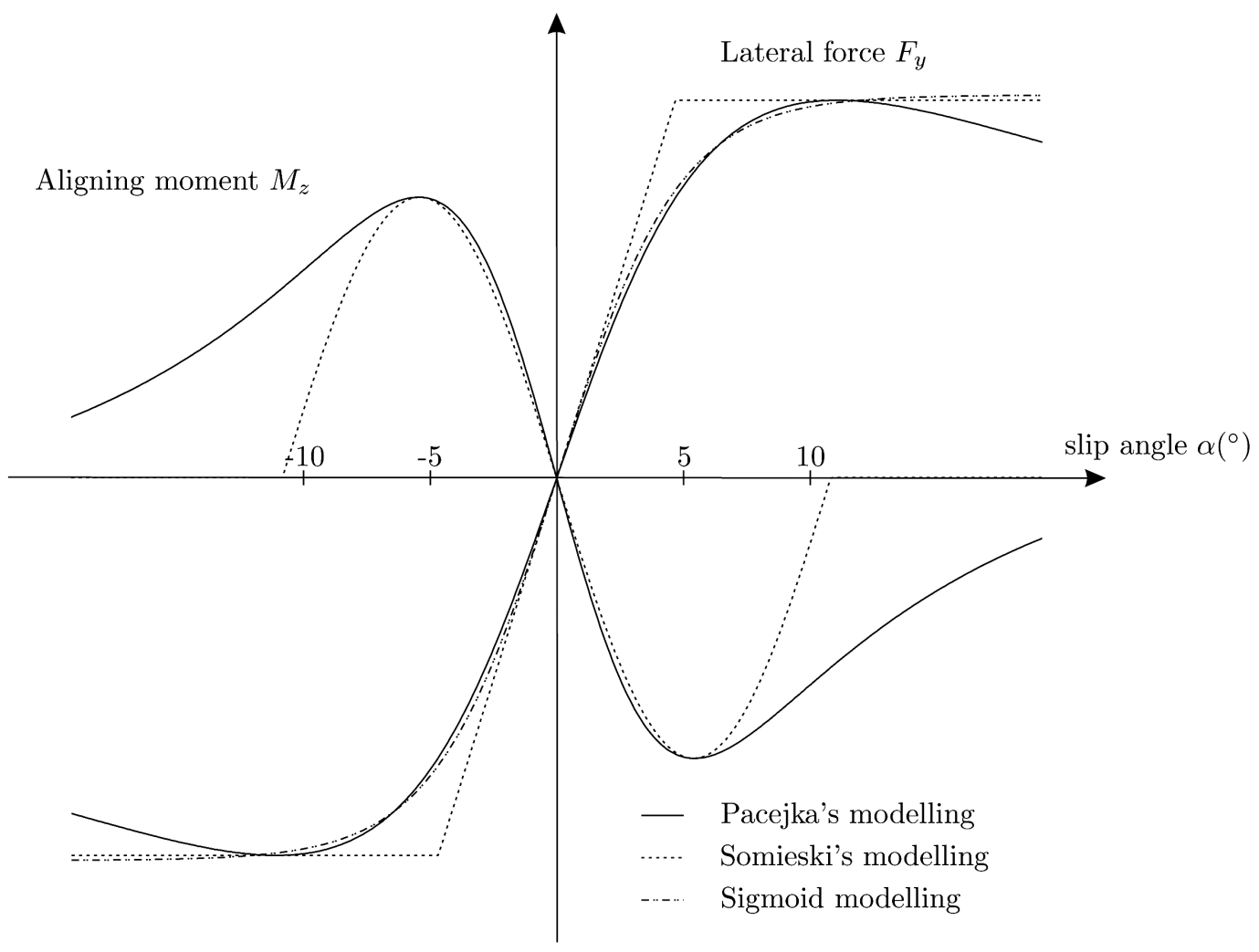

Fig. 2. General shapes of tires contributions $\left(M_{z}\right.$ and $\left.F_{y}\right)$.

of the lateral force could be improved (see Fig. 2). In this study, the piecewise linear curve is replaced by a sigmoid function (see (8)), in order to keep a simple expression of the model devoted to control-oriented applications. As can be noticed in Fig. 2, this model provides a better approximation of the lateral force: the proposed modeling based on a sigmoid function is continuous and approximates in a better way the corresponding Pacejka model which has been used as a reference model in this study for the validation of the sigmoid representation. Indeed, this new representation extends the validity domain of the lateral force $F_{y}$ to a higher tire slip angle $\left(\alpha=10^{\circ}\right)$ by taking account of the rounded shape of the curve around the maximum of the slip angle.

$$
F_{y}=c_{F_{\alpha}} F_{z} \delta\left(\frac{2}{1+e^{-\frac{2}{\delta} \alpha}}-1\right)
$$

\subsection{State Space Representation}

A state space representation of the NLG model is needed to design the adaptive damping controllers. By choosing the state variables $x_{1}=\psi_{w}, x_{2}=\dot{\psi}_{w}, x_{3}=y_{l}, x_{4}=\psi_{a}$, $x_{5}=\dot{\psi}_{a}$ and considering the control torque $u$, the nonlinear dynamics presented above can be expressed as:

$$
\left\{\begin{array}{l}
\dot{x}_{1}=x_{2} \\
\dot{x}_{2}=\frac{k_{s}\left(x_{4}-x_{1}\right)}{J_{z}}+\frac{k_{d}\left(x_{5}-x_{2}\right)}{J_{z}}+f_{1}\left(x_{3}\right)+f_{2}\left(x_{2}\right) \\
\dot{x}_{3}=v x_{1}+(e-a) x_{2}-\frac{v}{\sigma} x_{3} \\
\dot{x}_{4}=x_{5} \\
\dot{x}_{5}=-\frac{B_{a} x_{5}}{J_{a}}-\frac{k_{s}\left(x_{4}-x_{1}\right)}{J_{a}}-\frac{k_{d}\left(x_{5}-x_{2}\right)}{J_{a}}+\frac{1}{J_{a}} u
\end{array}\right.
$$

where:

$$
\begin{aligned}
& f_{1}\left(x_{3}\right)=\frac{M_{3}(\alpha)}{J_{z}}=\frac{M_{3}\left(y_{l} / \sigma\right)}{J_{z}} \\
& f_{2}\left(x_{2}\right)=\frac{M_{4}\left(\dot{\psi}_{w} / v\right)}{J_{z}}
\end{aligned}
$$

The output of the system is $y=\psi_{w}=x_{1}$. Hence the third derivative of the output:

$$
y^{(3)}=\frac{k_{s}\left(\dot{x}_{4}-\dot{x}_{1}\right)}{J_{z}}+\frac{k_{d}\left(\dot{x}_{5}-\dot{x}_{2}\right)}{J_{z}}+\dot{f}_{1}\left(x_{3}\right) \dot{x}_{3}+\dot{f}_{2}\left(x_{2}\right) \dot{x}_{2}
$$


Replacing the derivatives of the state variables (9) by (12), it is obvious that the input $u$ appears on the right hand side of the result. This means that the system has the relative degree $r=3$, and can be described by the following equation:

$$
y^{(3)}=a(\mathbf{x})+b(\mathbf{x}) u
$$

where $\mathbf{x}=\left[x_{1}, x_{2}, \ldots, x_{5}\right]^{T}$ is the system's state vector, $a(\mathbf{x})$ and $b(\mathbf{x})$ are nonlinear smooth functions. The explicit descriptions of these two nonlinear functions can be obtained after some mathematical manipulations. However, even if the exact expressions are calculated, they might not accurately describe the dynamics of the system when it is operating, because of time-varying parameters such as the vertical force or tire characteristics. For this reason, $a(\mathbf{x})$ and $b(\mathbf{x})$ are considered as unknown functions, and the adaptive control theory is adopted to cope with this uncertainty.

\subsection{Shimmy Test Scenarios: Open Loop Results}

In order to be comparable with previously published work on shimmy analysis, the proposed model (except for the actuator characteristics) has been configured with the parameters presented in [22] and summarized in the following table:

\begin{tabular}{|c|c|c|}
\hline Parameter & Value & Unit \\
\hline$v$ & $0 \ldots 80$ & $\mathrm{~m} / \mathrm{s}$ \\
\hline$a$ & 0.1 & $m$ \\
\hline$e$ & 0.1 & $m$ \\
\hline $\begin{array}{l}c_{F \alpha} \\
\delta\end{array}$ & $\begin{array}{l}20 \\
5\end{array}$ & $\underset{\circ}{\mathrm{rad}^{-1}}$ \\
\hline $\begin{array}{l}c_{M \alpha} \\
\alpha_{g}\end{array}$ & $\begin{array}{l}-2 \\
10\end{array}$ & $\underset{\circ}{m . r a d}{ }^{-1}$ \\
\hline$J_{a}$ & 0.1 & kg. $m^{2}$ \\
\hline$J_{z}$ & 1 & kg. $m^{2}$ \\
\hline$B_{a}$ & 0.1 & N.m.rad ${ }^{-1} . s$ \\
\hline$F_{z}$ & 9000 & $N$ \\
\hline$k_{S}$ & 100000 & N.m.rad ${ }^{-1}$ \\
\hline$k_{d}$ & 10 & N.m.rad ${ }^{-1} . s$ \\
\hline$\kappa$ & -270 & $N \cdot m^{2} \cdot r a d^{-1}$ \\
\hline$\sigma$ & 0.3 & $m$ \\
\hline
\end{tabular}

These parameters correspond to the NLG of a commercial civil aircraft.

To illustrate the performance of the model composed of the electrical actuator, the NLG and the tire/road interface, three different test scenarios have been chosen. In the aeronautical domain, these test cases describing real situations help to test the behavior of the NLG. For all the three proposed cases, the turning tube is kept at zero position and a perturbation is applied directly on the tire. The case studies that have been selected are:
- Scenario 1: Constant ground speed, pulse disturbance

The aircraft is supposed to have a forward ground speed of $80 \mathrm{~m} / \mathrm{s}$, the disturbance is a torque pulse of $1000 \mathrm{~N} . \mathrm{m}$ for $0.1 s$ and directly acts on the vertical axis at the wheel level. This test actually corresponds to a tire damage scenario.

- Scenario 2: Constant ground speed, rough runway

The purpose of this test is to investigate the effect of the roughness of the runway while the aircraft is running at a maximum speed of $80 \mathrm{~m} / \mathrm{s}$. This influence is modeled by a random disturbance which is a white noise with zero mean and a standard deviation of $100 N . m$.

- Scenario 3: Varying ground speed, rough runway

In this scenario, the same test as in scenario 2 is performed, with a varying forward velocity of the aircraft: $v$ changes from 10 to $80 \mathrm{~m} / \mathrm{s}$. This test is carried out because of the sensitivity of the shimmy phenomenon to the aircraft forward velocity [22].

The simulation of scenario 1 with the model described in Section 3.1 is illustrated in Fig. 3. The disturbance is applied on the tire at time $0.2 \mathrm{~s}$ for a duration of $0.1 \mathrm{~s}$. As can be seen in the figure, the NLG starts to oscillate, i.e. shimmy appears, as soon as the perturbation is applied on the tire. Then, the amplitude of the oscillations starts growing with time and the system becomes unstable.

Scenario 2 is illustrated in Fig. 4. The disturbance, representing a high roughness of the runway, is applied on the tire at the beginning of the simulation and the aircraft runs at $80 \mathrm{~m} / \mathrm{s}$. As can be seen in the figure, the NLG slowly starts to oscillate and after a while, as in scenario 1 , becomes unstable in the same way.

Concerning these two scenarios, the results are those obtained for a simulation of $1 \mathrm{~s}$.

Scenario 3 is illustrated in Fig. 5. The aircraft forward velocity is a critical parameter of the shimmy phenomenon and this scenario aims at illustrating this aspect. During landing and takeoff, the aircraft ground velocity varies between 0 and $80 \mathrm{~m} / \mathrm{s}$ and this scenario shows the impact of this varying parameter on the behavior of the NLG; the simulation is performed on $15 s$. At low speed (under $25 \mathrm{~m} / \mathrm{s}$ ), the system is stable whereas it becomes unstable and shimmy appears when the forward velocity of the aircraft is higher than $25 \mathrm{~m} / \mathrm{s}$.

These 3 scenarios show a shimmy phenomenon characterized by a natural frequency around $50 \mathrm{~Hz}$ and an increasing amplitude up to $20^{\circ}$. These results are in adequacy with the ones which can be found in the literature. It must be underlined that such a situation could generate malfunctioning and/or severe damages of the landing gear. That is why damping solutions are required. 

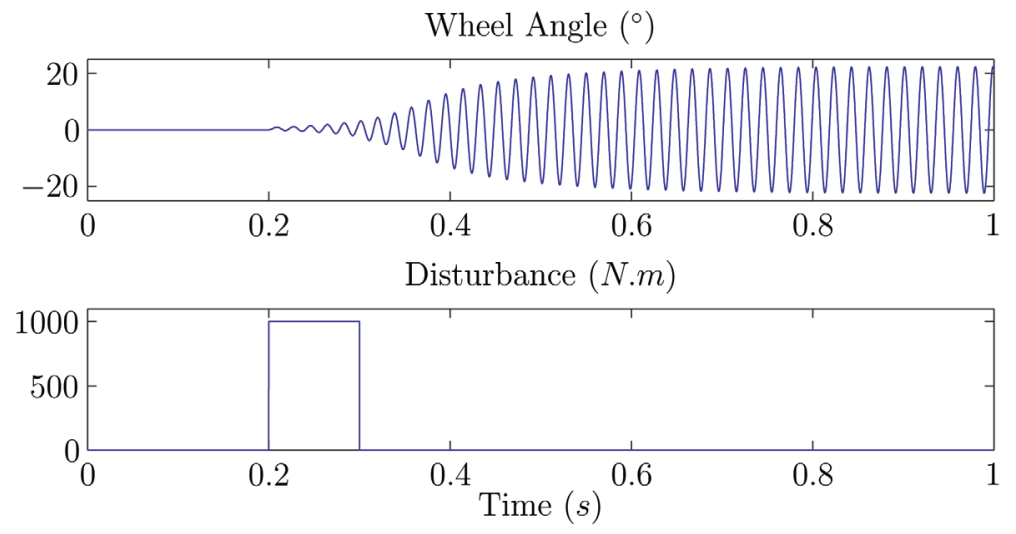

Fig. 3. Shimmy caused by a tire damage.
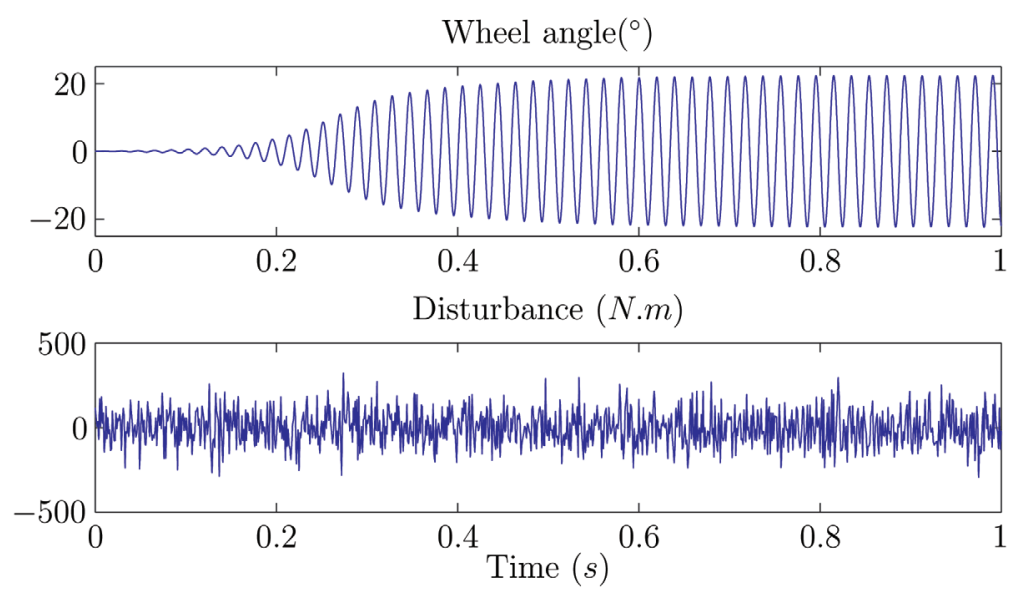

Fig. 4. Shimmy caused by a rough runway.

\section{State Feedback Active Shimmy Damping}

\subsection{Takagi-Sugeno Fuzzy Systems}

Fuzzy systems are one of the most appropriate approaches to expert knowledge modeling. This solution is generally used for plants which are mathematically difficult to model accurately and where the experience of an operator is needed. In the NLG model presented previously, it is difficult to accurately describe the tire/road interface, the importance of weather conditions, the influence of varying load, etc. This is why the fuzzy theory is necessary. The capability of approximating a function helps to cope with these uncertainties and will be used differently in the direct and indirect algorithms. For the indirect adaptive solution, the fuzzy system is used to estimate the NLG dynamics before computing the control input while the direct fuzzy adaptive solution directly allows the approximation of the control input.

Several standard fuzzy systems are described in the literature. In particular, the two algorithms developed in this paper are based on the Takagi-Sugeno fuzzy system such that the $p$ fuzzy rules have the following form:

$$
\begin{aligned}
R_{m_{(m=1 \cdots p)}:} \quad \text { If }\left(x_{1} \text { is } F_{1}^{1} \text { and } \cdots \text { and } x_{n} \text { is } F_{1}^{n}\right) \\
\text { Then } y^{m}=\theta_{m}
\end{aligned}
$$

where $F_{i}^{j}$ is the $i$ th fuzzy set (a part of the discourse universe) for the state variable $j\left(i=1 \ldots k\right.$ for the state $x_{1}$ or $i=1 \ldots l$ for the state $\left.x_{n}, j=1 \ldots n\right)$. For each fuzzy set $F_{i}^{j}$, a membership function $\mu$ such that $\mu_{F_{i}^{j}} \in[0,1]$ is defined. Several membership functions can be found in the literature and Gaussian membership functions are designed for this study. These functions are specified with a "center" $c$ and a "width" $\sigma$ such that:

$$
\mu(x)=\exp \left(-\left(\frac{x-c}{\sigma}\right)^{2}\right)
$$

Furthermore, the fuzzy Cartesian product is used to implement the "and" operation present in the fuzzy rule. Finally, 

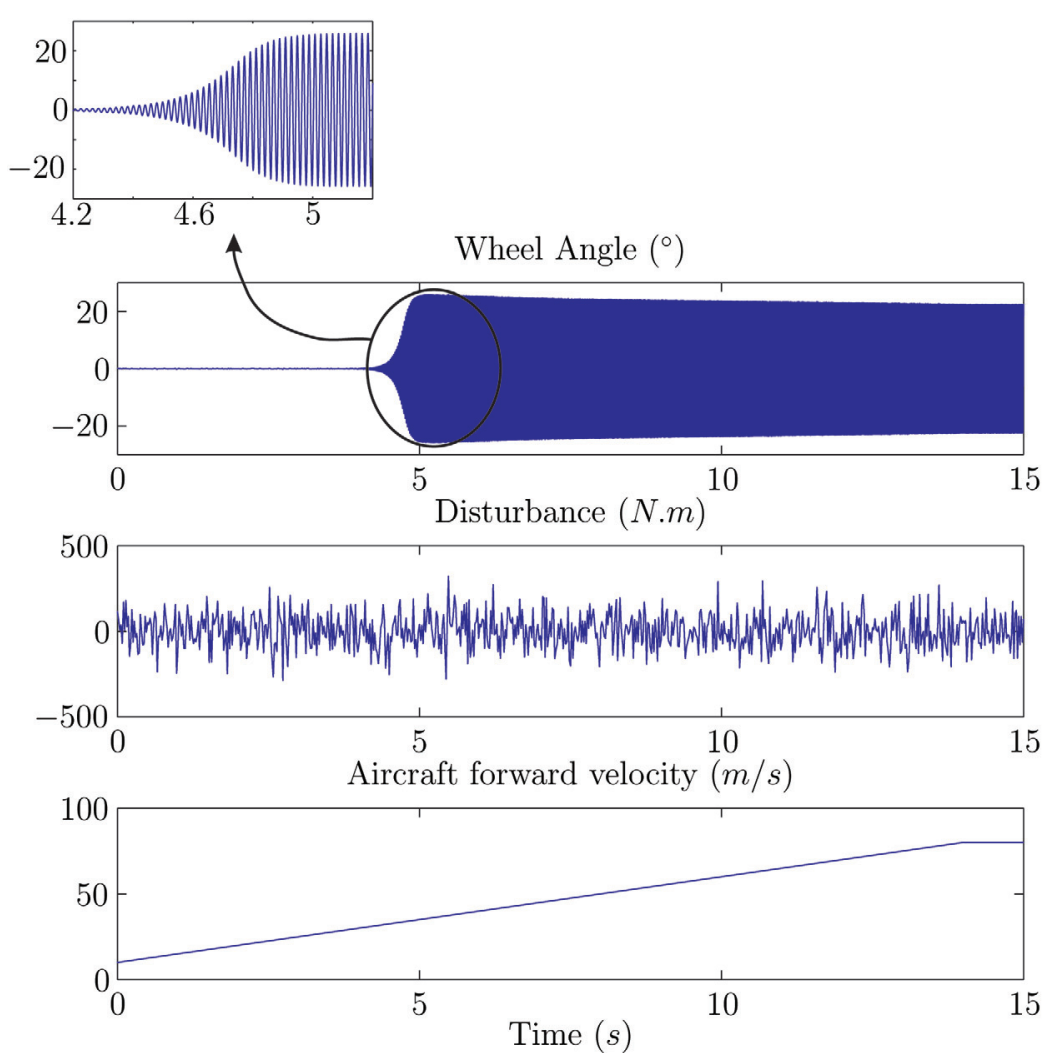

Fig. 5. Impact of the forward velocity.

a weighted average method is used for the defuzzification and the output $y$ of the fuzzy system is:

$$
y=\frac{\sum_{i=1}^{p} \theta_{i} \mu_{i}}{\sum_{i=1}^{p} \mu_{i}}=\theta^{T} \varsigma
$$

where $\mu_{i}:=\mu_{F_{k}^{1} \times F_{l}^{2} \times \cdots \times F_{m}^{n}}\left(x_{1}, x_{2}, \ldots, x_{n}\right)$ is the membership function of the $i$ th rule, $\theta^{T}:=\left[\theta_{1}, \ldots, \theta_{p}\right]$ and $\varsigma^{T}:=\left[\mu_{1} \ldots \mu_{p}\right] /\left[\sum_{i=1}^{p} \mu_{i}\right]$.

The design of fuzzy systems can be performed by the use of two main methods: an empirical method and an automatic method. The first method gives the possibility to manually choose the number of rules, the parameters of the membership function and the outputs of the fuzzy system. Based on a trial and error process with experimental tests, satisfying fuzzy system parameters can be obtained. This kind of method is suitable when the size of the fuzzy system is "sufficiently" low and when the input variable space can be easily fitted. Nevertheless, this method has the drawback to generally define more terms than are needed. The second method consists in searching optimal parameters of the fuzzy system. In [29], a genetic algorithm gives the possibility to optimally define the fuzzy system parameters. This method permits to improve the performances of the fuzzy system but a large number of training samples are required. Section 4.4 describes how the fuzzy systems have been tuned (number of rules, membership functions, ...).

\subsection{Indirect Fuzzy Adaptive Control}

A simplified version of the indirect fuzzy adaptive solution from [23] is developed here. The particularity of the proposed NLG model appears when (12) is developed (using (9) and (13)). Indeed, in this formulation, the contribution $b(\mathbf{x})$ does not explicitly depend on the state variables but only on structural parameters of the NLG model:

$$
B=\frac{k_{d}}{J_{a} J_{z}}
$$

In this study, $k_{d}, J_{a}$ and $J_{z}$ will be considered constant in the working domain of the controllers. This simplification aims at reducing the number of required fuzzy systems necessary to estimate the plant dynamics, since only $a(\mathbf{x})$ must be estimated. Finally, a simplified indirect adaptive controller is obtained. Considering this aspect, the indirect adaptive control solution presented in [23] is simplified 


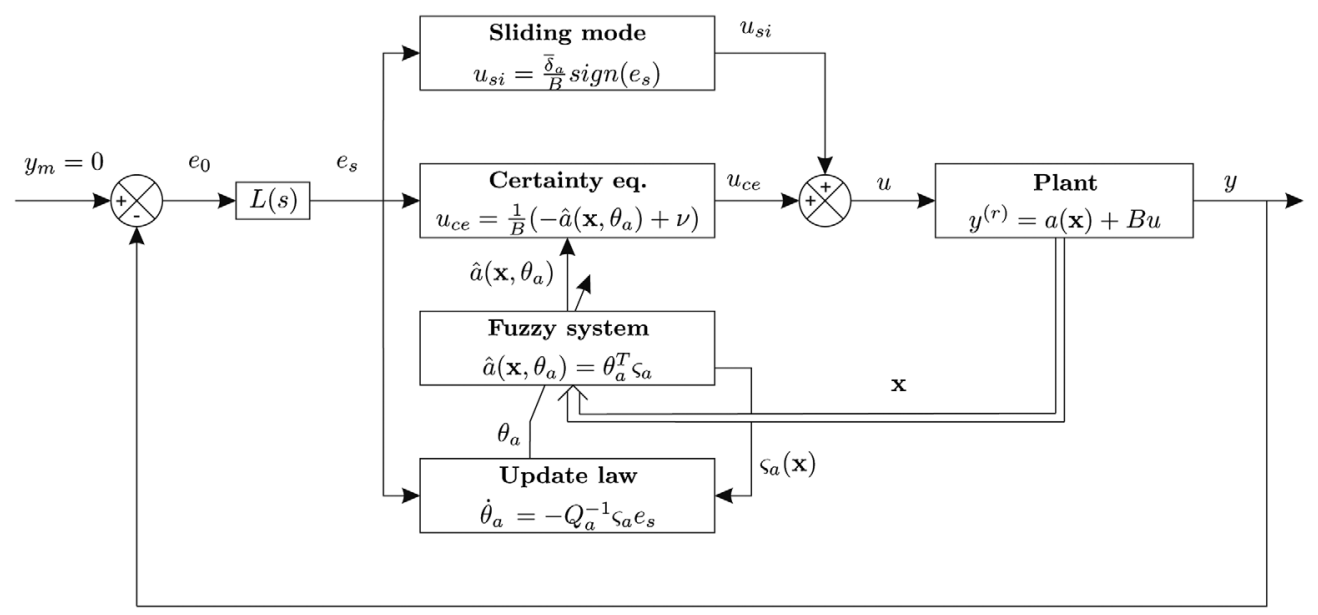

Fig. 6. Indirect fuzzy adaptive control scheme.

and the following nonlinear system is obtained:

$$
y^{(r)}=a(\mathbf{x})+B u
$$

The indirect adaptive feedback controller developed in this paper is represented in Fig. 6 .

The plant is represented by (18). In this strategy, the states of the system are used to estimate the dynamics of the NLG $\hat{a}(\mathbf{x})$ through a fuzzy system in which the output of the rules $\theta_{a}$ are adapted using an update law. The control signal $u$ is composed of the certainty equivalence control term $u_{c e}$ based on the estimated system dynamics $\hat{a}(\mathbf{x})$ and the sliding mode term $u_{s i}$. The latter helps to overcome the estimation error of the fuzzy system.

The certainty equivalence control term is used to estimate the feedback linearization control term ; it is defined such that the output of the system follows the reference $y_{m}$ and such that the error $e_{0}=y_{m}-y$ decreases exponentially:

$$
u_{c e}=\frac{1}{B}\left(-\hat{a}\left(\mathbf{x}, \theta_{a}\right)+v\right)
$$

where $v(t):=y_{m}^{(r)}+\eta e_{s}+\bar{e}_{s}$ and $\bar{e}_{s}:=\dot{e}_{s}-e_{o}^{(r)}$. The tracking error $e_{s}$ is defined as $e_{s}:=k^{T} e$ with $e:=$ $\left[e_{0} \dot{e}_{0} \ldots e_{0}^{(r-1)}\right], k:=\left[k_{0} \ldots k_{r-2} 1\right]$.

The elements of $k$ are chosen such that $L(s):=s^{r-1}+$ $k_{r-2} s^{r-2}+\ldots+k_{1} s+k_{0}$ is Hurwitz (see Section 4.4).

In this indirect fuzzy adaptive algorithm, $a(\mathbf{x})$ is an unknown nonlinear function which must be estimated by the fuzzy system using (20), to calculate the feedback linearization control law:

$$
\hat{a}\left(\mathbf{x}, \theta_{a}\right)=\theta_{a}^{T} \varsigma_{a}
$$

where $\theta_{a}^{T}:=\left[\theta_{a_{1}} \ldots \theta_{a_{p}}\right]$ and $\varsigma_{a}^{T}:=\left[\mu_{a_{1}} \ldots \mu_{a_{p}}\right] /$ $\left[\sum_{i=1}^{p} \mu_{a_{i}}\right]$. Moreover, the unknown function $a(\mathbf{x})$ can be formulated as follows:

$$
a(\mathbf{x})=\theta_{a}^{* T} \varsigma_{a}+\delta_{a}(\mathbf{x})
$$

where $\delta_{a}(\mathbf{x})$ is the optimal approximation error of $a(\mathbf{x})$ by the fuzzy system and $\theta_{a}^{*}$ is the best value of the parameter $\theta_{a}$ :

$$
\theta_{a}^{*}:=\arg \min _{\theta_{a}}\left[\sup _{\mathbf{x}}\left|\theta_{a}^{T} \varsigma_{a}-a(\mathbf{x})\right|\right]
$$

It is thus possible to prove that the fuzzy system can approximate a smooth nonlinear function with arbitrary small error if the number of fuzzy rules is large enough [12]. The parameter error vector $\tilde{\theta}_{a}(t)$ represents the difference between the current estimated parameter and the best value of this parameter and is defined by:

$$
\tilde{\theta}_{a}(t)=\theta_{a}(t)-\theta_{a}^{*}
$$

The estimation of the function $\hat{a}\left(\mathbf{x}, \theta_{a}\right)$ in (20) needs to be updated in order to follow the system dynamics and the variations induced by external perturbations. Then, the parameter $\theta_{a}$ of the fuzzy system is updated such that:

$$
\dot{\theta}_{a}=-Q_{a}^{-1} \varsigma_{a} e_{s}
$$

with $Q_{a} \in \Re^{p \times p}$ a square positive semidefinite matrix which helps to vary the adaptation speed of the fuzzy system. The sliding mode control term $u_{s i}$ has been chosen to ensure the stability of the control law and is expressed by:

$$
u_{s i}=\frac{\bar{\delta}_{a}}{B} \operatorname{sign}\left(e_{s}\right)
$$

The constant $\bar{\delta}_{a} \in \Re$ is defined such that the estimation error $\delta_{a}(\mathbf{x})$ is bounded:

$$
\left|\delta_{a}(\mathbf{x})\right| \leq \bar{\delta}_{a}
$$


In (26), $\bar{\delta}_{a}$ represents a known bound of the error estimation related to the fuzzy system. Since the fuzzy system is a universal approximator, it is considered that $\delta_{a}(\mathbf{x})$ remains arbitrarily small, if an appropriate fuzzy system with an arbitrarily large number of rules is chosen. Finally, $\left|\delta_{a}(\mathbf{x})\right|$ can be bounded by $\bar{\delta}_{a}$ and this term is used in this control law to counteract the modeling error between the real nonlinear function $a(\mathbf{x})$ and its estimate $\hat{a}\left(\mathbf{x}, \theta_{a}\right)$.

Aeronautical constraints are very strict and it is fundamental to ensure that the control solution is stable. The stability theorem and its proof for the indirect solution described initially in [23] and applied to the proposed active shimmy controller in [21] is formulated hereafter.

Theorem 4.1: Stability and tracking error results: Considering the system defined in (18), the control signal defined in (19), (25) and assuming the following:

- A1: the error estimation due to the fuzzy system is bounded (26).

It can be concluded that:

- C1: the plant output and its derivatives up to $(r-1)$ order are bounded.

- C2: the control signal is bounded.

- C3: the output error e $e_{0}$ will converge to zero.

Proof: The r-derivative of the output error can be written as:

$$
\begin{aligned}
e_{0}^{(r)} & =y_{m}^{(r)}-y^{(r)} \\
& =y_{m}^{(r)}-a(\mathbf{x})-B\left(u_{c e}+u_{s i}\right) \\
& =-a(\mathbf{x})+\hat{a}\left(\mathbf{x}, \theta_{a}\right)-\eta e_{s}-\bar{e}_{s}-B u_{s i}
\end{aligned}
$$

The tracking error equation becomes:

$$
\begin{aligned}
\dot{e}_{s}+\eta e_{s} & =-B u_{s i}-a(\mathbf{x})+\hat{a}\left(\mathbf{x}, \theta_{a}\right) \\
& =-B u_{s i}+\left(\phi_{a}^{T} \zeta_{a}-\delta_{a}(\mathbf{x})\right)
\end{aligned}
$$

Considering the Lyapunov function candidate:

$$
V=\frac{1}{2} e_{s}^{2}+\frac{1}{2} \tilde{\theta}_{a}^{T} Q_{a} \tilde{\theta}_{a}
$$

where $Q_{a} \in \Re^{d \times d}\left(d=\operatorname{dim}\left(\phi_{a}\right)\right)$ is a positive definite matrix. Differentiating $V(t)$ with respect to time leads to:

$$
\dot{V}=-\eta e_{s}^{2}-B u_{s i} e_{s}+\left(\hat{a}\left(\mathbf{x}, \theta_{a}\right)-a(\mathbf{x})\right) e_{s}+\tilde{\theta}_{a}^{T} Q_{a} \dot{\tilde{\theta}}_{a}
$$

Considering equations (23) and (24), the derivative of the parameter error vector becomes:

$$
\dot{\tilde{\theta}}_{a}=\dot{\theta}_{a}
$$

Consequently $\dot{V}$ becomes:

$$
\dot{V}=-\eta e_{s}^{2}-B u_{s i} e_{s}+(\hat{a}(\mathbf{x})-a(\mathbf{x})) e_{s}-\tilde{\theta}_{a}^{T} \varsigma_{a} e_{s}
$$

Equations (21) and (23) enable the following simplifica-
t1on:

$$
\dot{V}=-\eta e_{s}^{2}-B u_{s i} e_{s}+\left(\tilde{\theta}_{a}^{T} \varsigma_{a}-\delta_{a}(\mathbf{x})\right) e_{s}-\tilde{\theta}_{a}^{T} \varsigma_{a} e_{s}
$$

Now the assumption $\mathrm{A} 1$ and the definition of $u_{s i}$ (equation 25) allow to write:

$$
\begin{aligned}
\dot{V} & =-\eta e_{s}^{2}-\bar{\delta}_{a} \operatorname{sign}\left(e_{s}\right) e_{s}-\delta_{a}(\mathbf{x}) e_{s} \\
& \leq-\eta e_{s}^{2}-\bar{\delta}_{a} \operatorname{sign}\left(e_{s}\right) e_{s}+\left|\delta_{a}(\mathbf{x})\right|\left|e_{s}\right| \\
& \leq-\eta e_{s}^{2} \leq 0
\end{aligned}
$$

This means that $V \in \mathscr{L}_{\infty}$. If $V \in \mathscr{L}_{\infty}$ then $e_{s} \in \mathscr{L}_{\infty}$ and $\tilde{\theta}_{a} \in \mathscr{L}_{\infty}$ by the definition of $V$.

If $G_{i}(s)$ is defined by:

$$
G_{i}(s)=\frac{s^{i}}{L(s)}
$$

for $i=0, \ldots, r-1$, it is simple to show that $G_{i}(s)$ is stable because $L(s)$ has its $r-1$ roots in the open left half plane. Thus, the error becomes:

$$
e_{0}^{(i)}=G_{i}(s) e_{s}
$$

with $e_{s} \in \mathcal{L}_{\infty}$.

Then, error $e_{0}^{(i)}$ is bounded for $i=0, \ldots, r-1$ and $e_{0}^{(k)}=$ $y_{m}^{(k)}-y^{(k)}$, so the conclusion is that $y(t), \ldots, y^{(r-1)}(t)$ are bounded.

As proven above, $\tilde{\theta}_{a}$ is bounded so the "certainty equivalence" control term is bounded. Moreover, the "sliding mode" control term is bounded. The conclusion is that $u$ is bounded.

If equation (34) is used:

$$
\int_{0}^{\infty} \eta e_{s}^{2} d t \leq-\int_{0}^{\infty} \dot{V} d t=V(0)-V(\infty)<\infty
$$

then $e_{s} \in \mathscr{L}_{2}$. Moreover previous considerations show that $\hat{a}(\mathbf{x}), \tilde{\theta}_{a}, \delta_{a}\left(\mathbf{x}, \theta_{a}\right)$ and $\zeta_{a}(\mathbf{x})$ are bounded. From (28) and the fact that $e_{s}$ and $u_{s i}$ are bounded, it is obvious that $\dot{e}_{s}$ is bounded. Thus, by Barbalat's Lemma, the tracking error $e_{s}$ will converge to zero and $e_{0}$ will converge to zero.

\subsection{Direct Fuzzy Adaptive Control}

The block diagram of the direct fuzzy adaptive controller, initially proposed in [23] is presented in Fig. 7. 


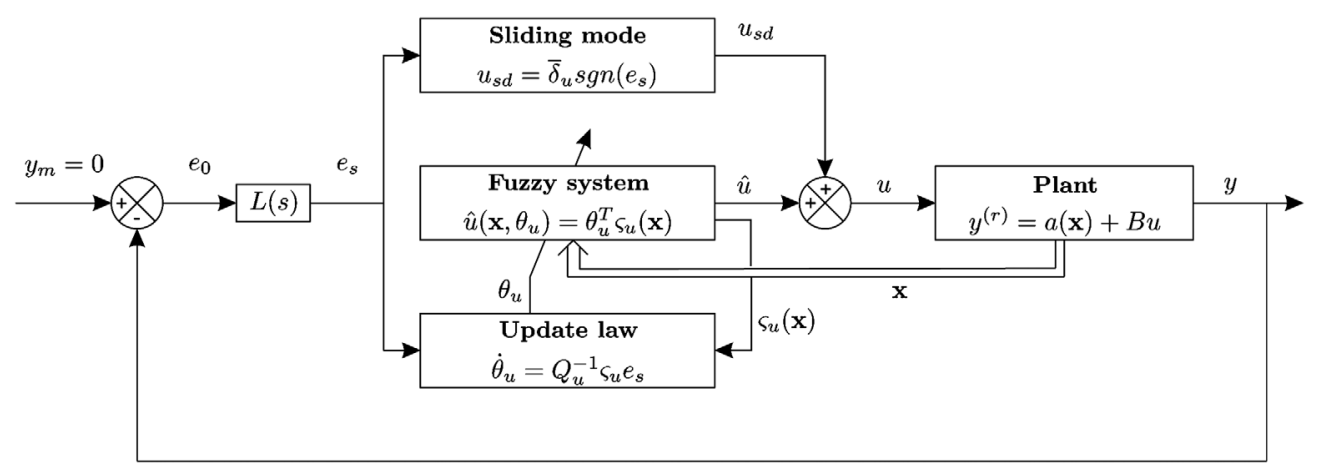

Fig. 7. Direct fuzzy adaptive control scheme.

In this strategy, there is no need to estimate the dynamics of the plant; the fuzzy system, adapted with an update law, is used to directly estimate the control signal $\hat{u}$. As is the case for the indirect solution, a sliding mode signal $u_{s d}$ helps to overcome the estimation error of the fuzzy system. The control signal $u$ is based on the estimated certainty equivalence control term $\hat{u}$ and the sliding mode term $u_{s d}$ such that:

$$
u=\hat{u}+u_{s d}
$$

On the assumption that function $a(\mathbf{x})$ and constant $B$ describing the system dynamics are unknown, the ideal feedback linear control law cannot be implemented. Instead, this control term is approximated by a universal approximator, here a fuzzy system, of the following form:

$$
\hat{u}\left(\mathbf{x}, \theta_{u}\right)=\theta_{u}^{T} \varsigma_{u}(\mathbf{x})
$$

Moreover, the unknown function $u(\mathbf{x})$ can be formulated as follows:

$$
u(\mathbf{x})=\theta_{u}^{* T} \varsigma_{u}+\delta_{u}(\mathbf{x})
$$

where $\delta_{u}(\mathbf{x})$ is the optimal approximation error of $u(\mathbf{x})$ by the fuzzy system and $\theta_{u}^{*}$ is the best value of the parameter $\theta_{u}$ :

$$
\theta_{u}^{*}:=\arg \min _{\theta_{u}}\left[\sup _{\mathbf{x}}\left|\theta_{u}^{T} \varsigma_{u}-u(\mathbf{x})\right|\right]
$$

The parameter error vector $\tilde{\theta}_{u}(t)$ represents the difference between the current estimated parameter and the best value of this parameter and is defined by:

$$
\tilde{\theta}_{u}(t)=\theta_{u}(t)-\theta_{u}^{*}
$$

The estimation of the function $\hat{u}$ needs to be updated to take account of the possible changes in the system behavior due to time varying parameters. Then, the following fuzzy system update law is chosen:

$$
\dot{\theta}_{u}=Q_{u}^{-1} \varsigma_{u} e_{s}
$$

with $Q_{u} \in \mathfrak{R}^{p \times p}$ a square positive semidefinite matrix.

The sliding mode control term which helps to overcome the approximation error of the fuzzy system and ensures the stability of the control loop, is expressed as:

$$
u_{s d}=\bar{\delta}_{u} \operatorname{sign}\left(e_{s}\right)
$$

The constant $\bar{\delta}_{u} \in \Re$ is defined such that:

$$
\left|\delta_{u}(\mathbf{x})\right| \leq \bar{\delta}_{u}
$$

It represents a known bound of the error estimation due to the fuzzy system.

The properties of the direct fuzzy adaptive controller are presented in the following theorem and the stability proof from [23] applied to the proposed controller in [10] is recalled here:

Theorem 4.2: Stability and tracking error results: Considering the system defined in (18), the control signal defined in (38), (39), (44) and assuming the following:

- A1: the error estimation due to the fuzzy system is bounded, so $\left|\delta_{u}(\boldsymbol{x})\right| \leq \bar{\delta}_{u}$.

It can be concluded that:

- C1: the plant output and its derivatives up to $(r-1)$ order are bounded.

- C2: the control signal is bounded.

- C3: the output error $e_{0}$ will converge to zero.

Proof. The $r$ th derivative of the output error is:

$$
\begin{aligned}
\dot{e}_{0}^{(r)} & =y_{m}^{(r)}-y^{(r)}=y_{m}^{(r)}-(a(\mathbf{x})+B u) \\
& =y_{m}^{(r)}-v-B\left(u-u^{*}\right)=-\bar{e}_{s}-\eta e_{s}-B\left(\hat{u}+u_{s d}-u^{*}\right) \\
& =-\bar{e}_{s}-\eta e_{s}-B \tilde{\theta}_{u}^{T} \varsigma_{u}+B \delta_{u}-B u_{s d}
\end{aligned}
$$

With $\bar{e}_{s}=\dot{e}_{s}-e_{0}^{(r)}$ the previous equation leads:

$$
\dot{e}_{s}+\eta e_{s}=-B \tilde{\theta}_{u}^{T} \varsigma_{u}+B \delta_{u}-B u_{s d}
$$


Considering the Lyapunov candidate function:

$$
V=\frac{1}{2 B} e_{s}^{2}+\frac{1}{2} \tilde{\theta}_{u}^{T} Q_{u} \tilde{\theta}_{u}
$$

where $Q_{u} \in \Re^{d \times d}\left(d=\operatorname{dim}\left(\phi_{u}\right)\right)$ is a positive definite matrix. Take the derivative of $V$ with respect to time and notice that $\dot{\tilde{\theta}}_{u}=\dot{\theta}_{u}$ imply:

$$
\begin{aligned}
\dot{V} & =\frac{1}{B} e_{s} \dot{e}_{s}+\tilde{\theta}_{u}^{T} Q_{u} \dot{\theta}_{u} \\
& =\frac{e_{s}}{B}\left(-\eta e_{s}-B \tilde{\theta}_{u}^{T} \varsigma_{u}+B \delta_{u}-B u_{s d}\right)+\tilde{\theta}_{u}^{T} Q_{u} \dot{\theta}_{u} \\
& =-\frac{\eta e_{s}^{2}}{B}-e_{s} u_{s d}+e_{s} \delta_{u}+\tilde{\theta}_{u}^{T}\left(Q_{u} \dot{\theta}_{u}-\varsigma_{u} e_{s}\right)
\end{aligned}
$$

The use of equation (43) enables to show that:

$$
\dot{V}=-\frac{\eta e_{s}^{2}}{B}-e_{s} u_{s d}+e_{s} \delta_{u}
$$

The definition of the sliding control term in equation (44) enables to write:

$$
\dot{V} \leq-\frac{\eta e_{s}^{2}}{B} \leq 0
$$

Since $V$ is a quadratic function and $\dot{V} \leq 0$ the control system is proved to be stable. It is clear that $V \in \mathscr{L}_{\infty}$, which implies $e_{s} \in \mathscr{L}_{\infty}$ and $\tilde{\theta}_{u} \in \mathscr{L}_{\infty}$. With $e_{s}$ bounded and the fact that $u_{s}$ bounded, the equation (47) gives $\dot{e}_{s} \in \mathscr{L}_{\infty}$.

If $G_{i}(s)$ is defined by:

$$
G_{i}(s)=\frac{s^{i}}{L(s)}
$$

for $i=0, \ldots, r-1$, it is simple to show that $G_{i}(s)$ is stable because $L(s)$ has its $r-1$ roots in the open left half plane. Thus, the error becomes:

$$
e_{0}^{(i)}=G_{i}(s) e_{s}
$$

Then, error $e_{0}^{(i)}$ is bounded for $i=0, \ldots, r-1$ and $e_{0}^{(k)}=$ $y_{m}^{(k)}-y^{(k)}$, so the conclusion is that $y(t), \ldots, y^{(r-1)}(t)$ are bounded.

From equation (50), the following is obtained:

$$
\int_{0}^{\infty} \eta e_{s}^{2} d t \leq-\int_{0}^{\infty} \dot{V} d t=V(0)-V(\infty)<\infty
$$

which implies that $e_{s} \in \mathscr{L}_{2}$. Thus, by Barbalat's Lemma, the tracking error $e_{s}$ will converge to zero and $e_{0}$ will converge to zero.

\subsection{Controller Design}

The active shimmy damping controllers for an aircraft NLG are designed on the indirect and direct adaptive fuzzy control algorithms discussed in the previous sections. Suppose that the aircraft moves straight forward, then the reference output $y_{m}$ corresponding to the desired wheel angle $\psi_{w}$ can be set to zero for the problem of shimmy damping. The following section details the design of the active damping controllers corresponding to the NLG parameters given in [22], and recalled in Section 3.3.

The outputs of the fuzzy systems are adaptively obtained based on an error minimization process, see (24) and (43). Then, only the number of fuzzy rules and the parameters of the membership functions are selected. The empirical method was adopted to obtain the parameters of the fuzzy system. Several reasons justify this choice:

- The fuzzy system is "sufficiently" simple (only 5 clearly defined inputs permit to obtain the output of fuzzy system),

- The parameters space of variation is known,

- The model of the system and the control law give the possibility to compare the function to be identified $(a(\mathbf{x})$ in the case of indirect solution) and the output of the fuzzy system $(\hat{a}(\mathbf{x}))$.

A heuristic method based on a trial and error technique has been adopted in this study. The number of required membership functions of the two fuzzy systems is obtained by simulation tests. A trade off between the number of membership functions and the performance of the fuzzy systems has been performed. As a conclusion, the fuzzy system obtained for the direct control solution is composed of 48 rules ( 3 (membership functions for $\left.\psi_{w}\right) \times 2$ (membership functions for $\psi_{a}$ ) $\times 2$ (membership functions for $\left.\dot{\psi}_{w}\right) \times 2$ (membership functions for $\left.\dot{\psi}_{a}\right) \times 2$ (membership functions for $\left.y_{l}\right)=48$ rules). Concerning the indirect control solution, the fuzzy system is composed of 72 rules ( 3 (membership functions for $\psi_{w}$ ) $\times 2$ (membership functions for $\left.\psi_{a}\right) \times 3$ (membership functions for $\left.\dot{\psi}_{w}\right) \times 2$ (membership functions for $\dot{\psi}_{a}$ ) $\times 2$ (membership functions for $\left.y_{l}\right)=72$ rules).

When two membership functions are used for a signal, the centers correspond to the two bounds of the varying space $-\overline{x_{i}}$ and $\overline{x_{i}}$. When three membership functions are required, the second center is placed in the middle such that $c=0$. Concerning the standard deviation $\sigma$, this parameter is chosen such that the membership function covers the whole variation space of the different parameters.

The NLG model considered is a $5^{\text {th }}$ order nonlinear system with a relative degree of 3 . In this case, the tracking error is $e_{s}(t)=\ddot{e}_{0}+k_{1} \dot{e}_{0}+k_{0} e_{0}$. The constants chosen for the indirect solution are $k_{0}=25$ and $k_{1}=7$, whereas the constants are equal to $k_{0}=50$ and $k_{1}=15$ 
for the direct solution. Parameter $k_{i}$ are chosen such that the polynomial has its roots in the open left half plane. The considered polynomial defines the tracking error $e_{s}$. The goal of the adaptive controller is to "learn" how to control the plant to drive $e_{s}$ to zero. As a conclusion, parameters $k_{i}$ give the possibility to define the convergence speed of the system, in terms of the error, its first derivative, etc.

The particularity of these algorithms is the use of a constant value for the control gain $B$. This constant, defined by the model parameters $k_{d}, J_{a}$ and $J_{z}$, is equal to 100 . The simulations showed that the maximum value of the estimates $\hat{a}(\mathbf{x})$ is approximately equal to 10,000 ; so, for the indirect solution, the error estimation is deliberately bounded by $10 \%$ of this maximum value $\left(\bar{\delta}_{a}=1,000\right)$. Concerning the direct solution, $\bar{\delta}_{u}$ is chosen to be small to limit the chattering phenomenon which may occur in the switching stabilizing control signal.

Moreover, the two adaptive control solutions are compared with a simple PID controller especially developed for this application. Different methods in the literature help to tune the parameters of a PID controller. For manual tuning, for example, the Ziegler-Nichols method or the Cohen-Coon method are well known, useful tuning rules. Based on these methods, the tuning of the PID parameters can be a delicate task and the PID controllers obtained are rarely tuned optimally. So, to overcome this constraint, a self-tuning method can be used to find the best values of the PID controller: here a neural network is used to self-adjust the PID parameters. In this approach, the gains $K_{p}, K_{i}$ and $K_{d}$ are determined in real time using a neural network. During the simulation tests, the adaptation law has been tuned to let the three parameters vary freely. Despite this important opportunity to change, the PID parameters rapidly converge to constant values. Therefore, it has been decided to fix the gains to the values obtained. Finally, the following tuning parameters $K_{p}=500, K_{i}=200$ and $K_{d}=95$ are obtained. This choice gives the possibility to obtain a common PID controller, without significant deterioration of simulation results. Indeed, the aim here is to compare a simple control solution classically used for real plants to the adaptive controllers developed for the NLG application described here.

\section{Control Performances (Simulation Results)}

To illustrate the performance of the proposed shimmy damping controllers, simulations have been carried out with the three previously described test scenarios. It is assumed, in these scenarios, that the bandwidth of the electrical actuator is sufficiently high (approximately $200 \mathrm{~Hz}$ ) to provide the simulated control torque. In this work, the mechanical transmission between the actuator and the turning tube is not considered. Moreover, a robustness analysis is performed. This latter aims at studying the impact of varying parameters on the stability and the performances of the closed-loop system.

\subsection{Scenario 1: Constant Ground Speed, Pulse Disturbance}

As explained in Section 3.3, this test corresponds to a tire damage simulation scenario. Fig. 8 and Fig. 9 show the response of the NLG with respectively the indirect and the direct active damping controllers.

It is obvious that no shimmy appears since the oscillations are rapidly and efficiently damped. However, as the figures reveal, there is a small bias angle in the time the disturbance is applied; the wheel only returns to its original zero position when the disturbance disappears. This behavior of the proposed active damping controllers is quite similar to that of current passive shimmy damping solutions: the main purpose of the controllers is not to drive the wheel, but to damp the shimmy oscillation. In fact, it is possible to choose the design parameters of the adaptive controllers so that the wheel angle remains close to its initial position $\left(0^{\circ}\right)$ even when the disturbance acts on the system, but in this case the control torque must be larger.

The two solutions give quite similar responses. In both cases, the oscillations are correctly damped and the maximum deviation of the wheel is lower than $1^{\circ}$ with a mean value of $0.5^{\circ}$.

Fig. 10 shows the response of the NLG with the PID controller in the same conditions. It is obvious that this solution does not give satisfactory results. On the one hand, the amplitude of the oscillations is important (more than $3^{\circ}$ ), while the two adaptive solutions help to obtain an amplitude below $1^{\circ}$. On the other hand, it can bee seen that the damping of the oscillations is practically non-existent, even after the applied disturbance. The response shows that the amplitude of the wheel angle is still important after $0.6 s$, while the two adaptive solutions help to negate the effect of the perturbation.

So far, the results obtained with these three control strategies have been compared, based on subjective criteria. The shape of the wheel angle $\psi_{w}$ helps to characterize the validity of the control solution. These subjective criteria used to validate or invalidate the control solution can be completed by defining an objective criteria based on the calculation of the damping ratio. The damping ratio $\zeta$ helps to obtain the properties of an oscillating signal, it describes how rapidly the oscillations decrease from one maximum or minimum to the next. The common formulation of the damping ratio uses two successive 
Wheel Angle $\left({ }^{\circ}\right)$
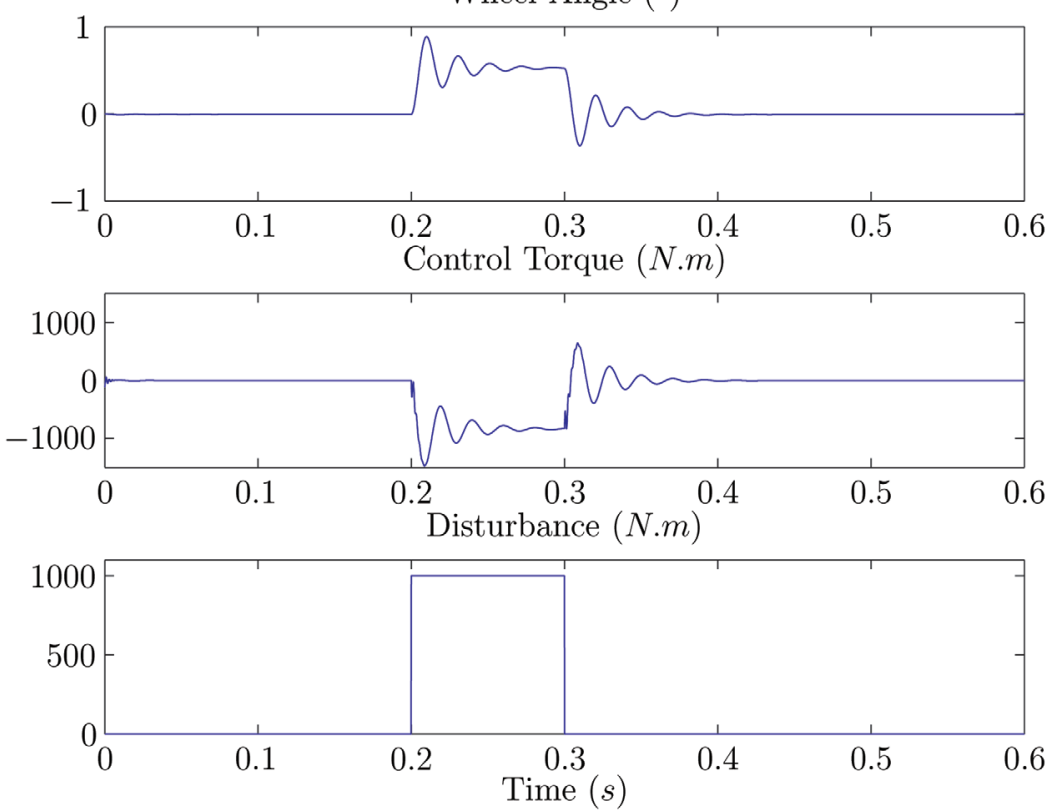

Fig. 8. Active shimmy damping Scenario 1 (Indirect solution).
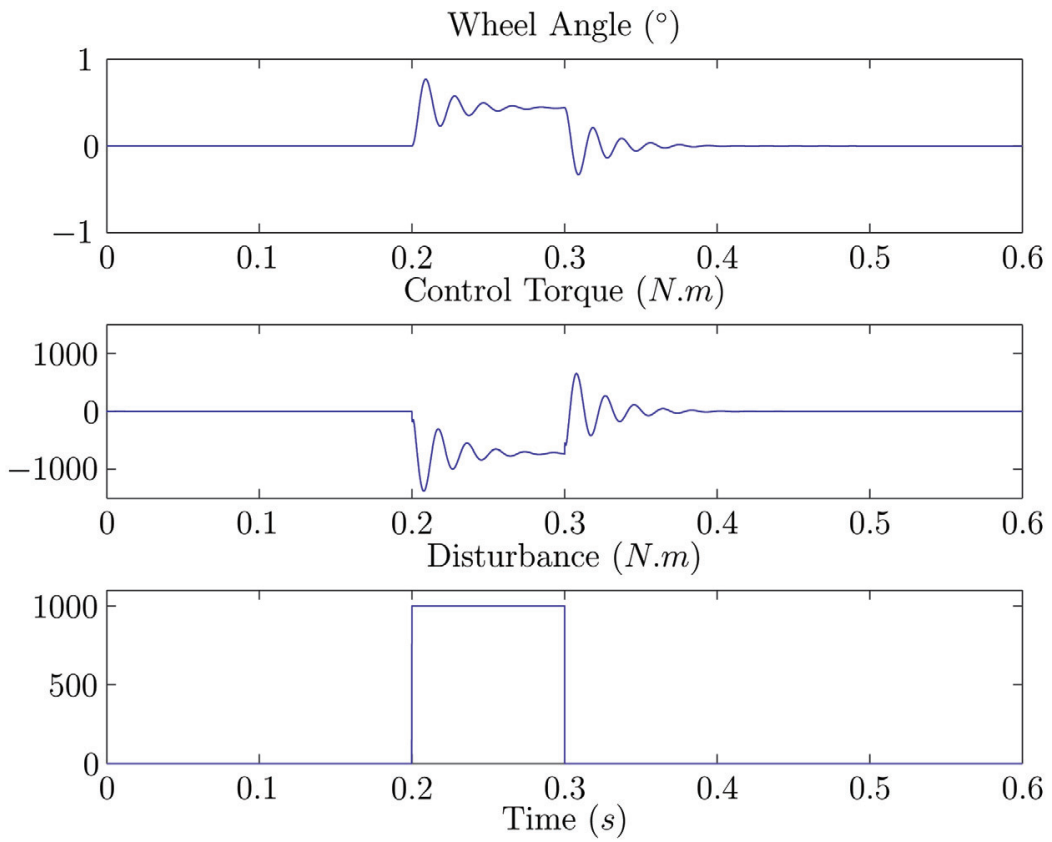

Fig. 9. Active shimmy damping Scenario 1 (Direct solution).

maximum or minimum values of the signal $\left(\psi_{w 1}\right.$ and $\left.\psi_{w 2}\right)$ and the logarithmic decrement $\Delta=\ln \left(\frac{\psi_{w 1}}{\psi_{w 2}}\right)$ such that:

$$
\zeta=\frac{\Delta / 2 \pi}{\sqrt{1+(\Delta / 2 \pi)^{2}}}
$$

This damping ratio formulation is used to compare the three proposed control solutions. When the damping ratio is close to zero the oscillations are slightly damped. The value of the damping ratio increases as soon as the oscillations are damped. The calculation is based on the two successive minima obtained after the disappearance of the 

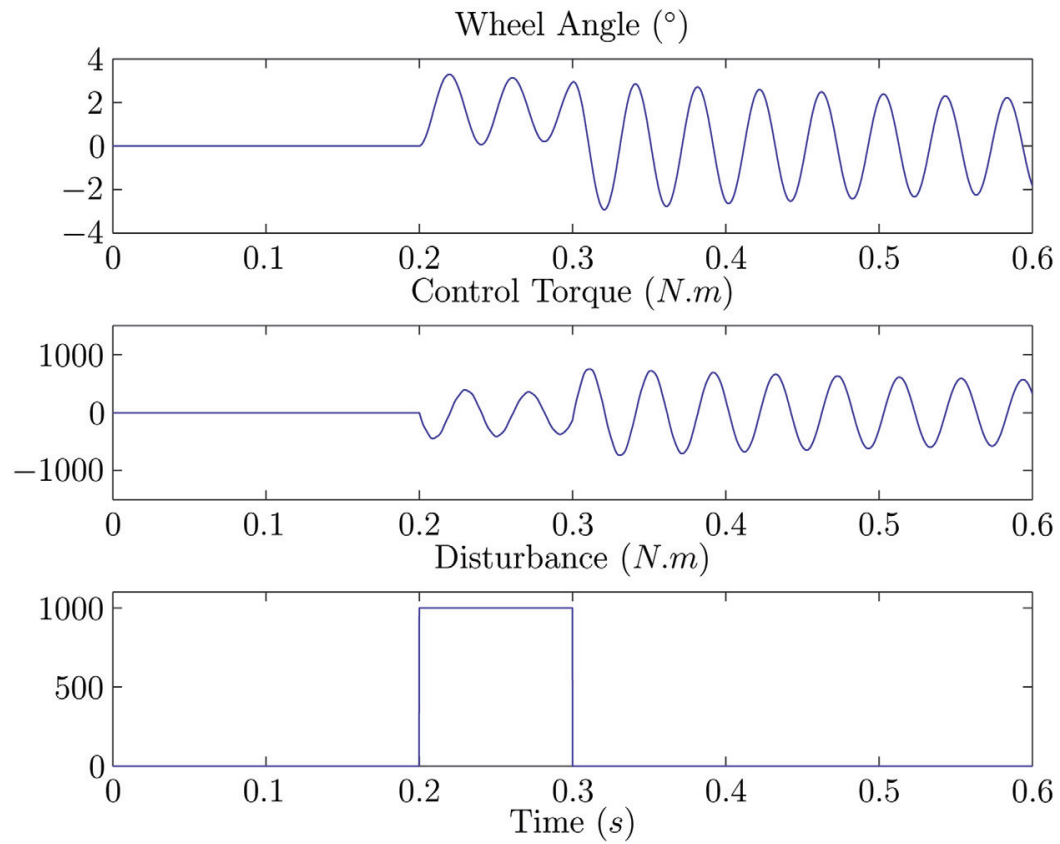

Fig. 10. Active shimmy damping Scenario 1 (PID solution).

perturbation. The following table presents the damping ratio obtained for the three control solutions:

\begin{tabular}{lccc}
\hline & $\begin{array}{c}\text { First } \\
\text { Minima } \psi_{w 1}\end{array}$ & $\begin{array}{c}\text { Second } \\
\text { Minima } \psi_{w 2}\end{array}$ & $\begin{array}{c}\text { Damping } \\
\text { Ratio } \zeta\end{array}$ \\
\hline $\begin{array}{c}\text { Indirect control } \\
\text { solution }\end{array}$ & -0.366 & -0.147 & $\mathbf{0 . 1 4 4}$ \\
$\begin{array}{c}\text { Direct control } \\
\text { solution }\end{array}$ & -0.332 & -0.138 & $\mathbf{0 . 1 3 8}$ \\
PID solution & -2.928 & -2.775 & $\mathbf{0 . 0 0 9}$ \\
\hline
\end{tabular}

This damping ratio calculation allows the validation of the subjective judgments mentioned previously. Indeed, the damping ratio of the indirect and direct control solutions, respectively equal to 0.144 and 0.138 , are close and these values are sufficiently important to characterize efficient damping. Nevertheless, for the PID solution, the damping ratio obtained is 0.009 . This value is close to zero, which corresponds to a low damping of the wheel angle $\psi_{w}$.

\subsection{Scenario 2: Constant Ground Speed, Rough Runway}

This scenario is performed to simulate the effects of the roughness of the runway on the NLG at high speed $(80 \mathrm{~m} / \mathrm{s})$. The results with the proposed active damping controllers are plotted in Fig. 11 and Fig. 12.

Even with important random perturbations and at a high forward velocity, shimmy does not occur.
Moreover, the variation of the wheel angle due to the high road imperfections, is very small (less than $0.2^{\circ}$ ). In practice, this small variation cannot cause any damage or malfunction to the NLG.

Fig. 13 shows the response of the NLG with the PID controller in the same context. The amplitude of the wheel angle with this random perturbation is not high, the PID controller is able to stabilize the NLG even if the phenomena observed may correspond to shimmy oscillations (approximately $50 \mathrm{~Hz}$ ). However, the two adaptive solutions give better results, because these solutions allow the amplitude of the wheel angle to fall under $0.2^{\circ}$ without periodic oscillations.

\subsection{Scenario 3: Varying Ground Speed, Rough Runway}

The aim of this test is to investigate the performance of the damping controllers when the system is under varying speed conditions. The forward velocity is a critical parameter in the shimmy phenomenon: the shimmy oscillations traditionally increase with the increasing velocity. In low speed conditions $(\leq 25 \mathrm{~m} / \mathrm{s})$, shimmy does not easily affect the NLG behavior [22]. So, this scenario helps to verify that the active controllers do not affect the system stability at low speed and that they damp the oscillations at high speed. Fig. 14 and Fig. 15 show the simulation results with the two active shimmy damping controllers in action.

Under the critical forward velocity of the aircraft $(\approx 25 \mathrm{~m} / \mathrm{s})$, the two controllers give similar results: 

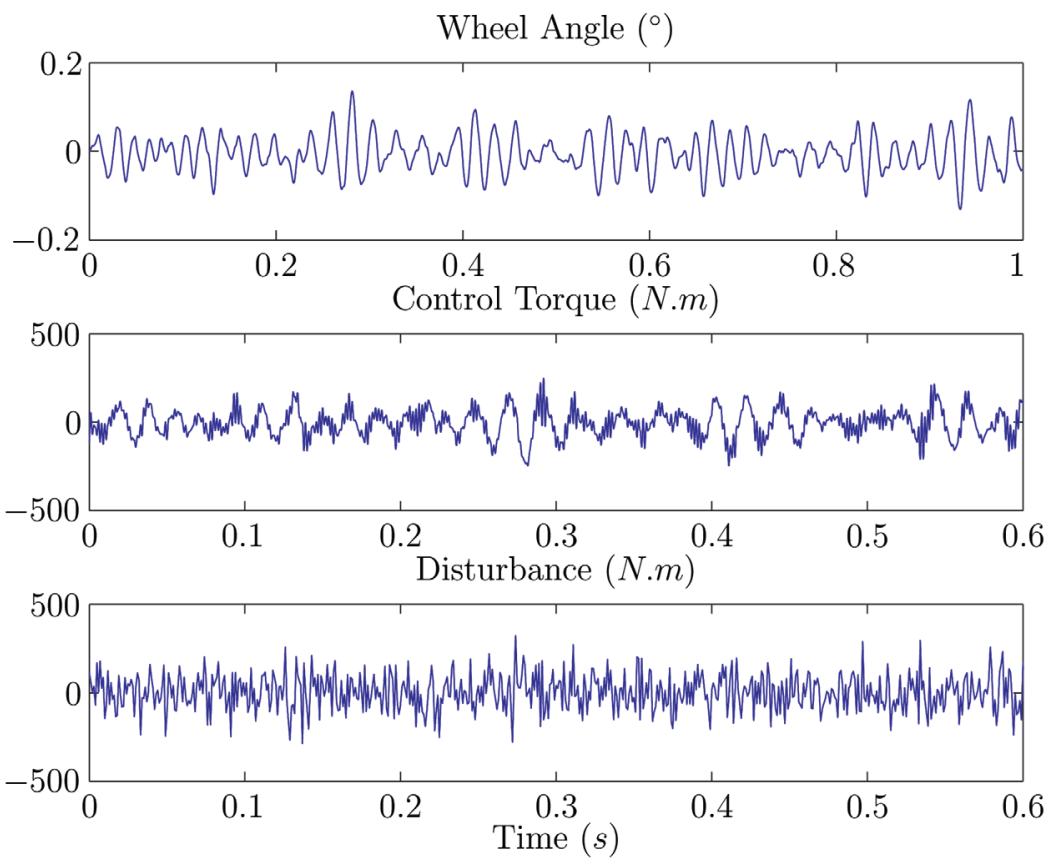

Fig. 11. Active shimmy damping Scenario 2 (Indirect solution).
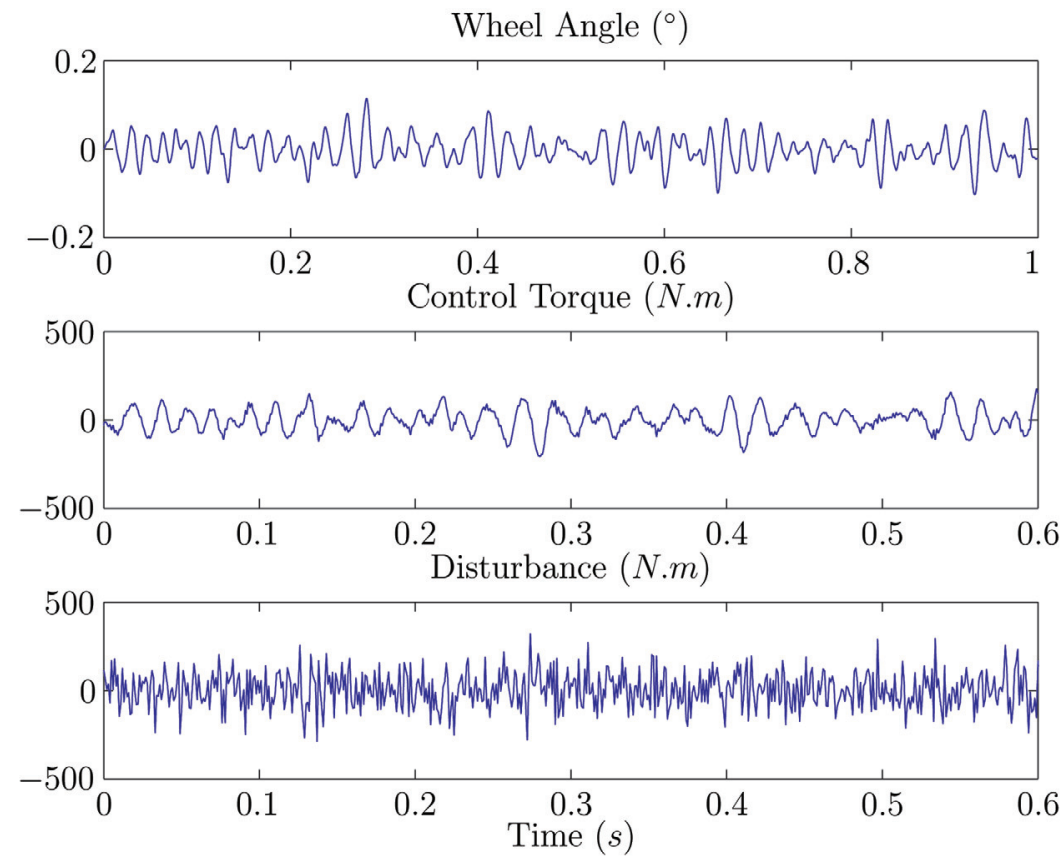

Fig. 12. Active shimmy damping Scenario 2 (Direct solution).

shimmy is not observed and the control laws do not destabilize the NLG. Beyond this critical speed, the shimmy oscillations which appear with the non-controlled plant (see Fig. 5) are actually damped.

Fig. 16 shows the response of the NLG with the PID controller. It is obvious that this solution does not give satisfactory results. First, it can be seen that shimmy oscillations appear rapidly under the critical forward velocity: the system becomes unstable because of the control loop. Secondly, the amplitude of the wheel angle with this random perturbation is very high (approximately $20^{\circ}$ at the beginning) but the controller finally manages 

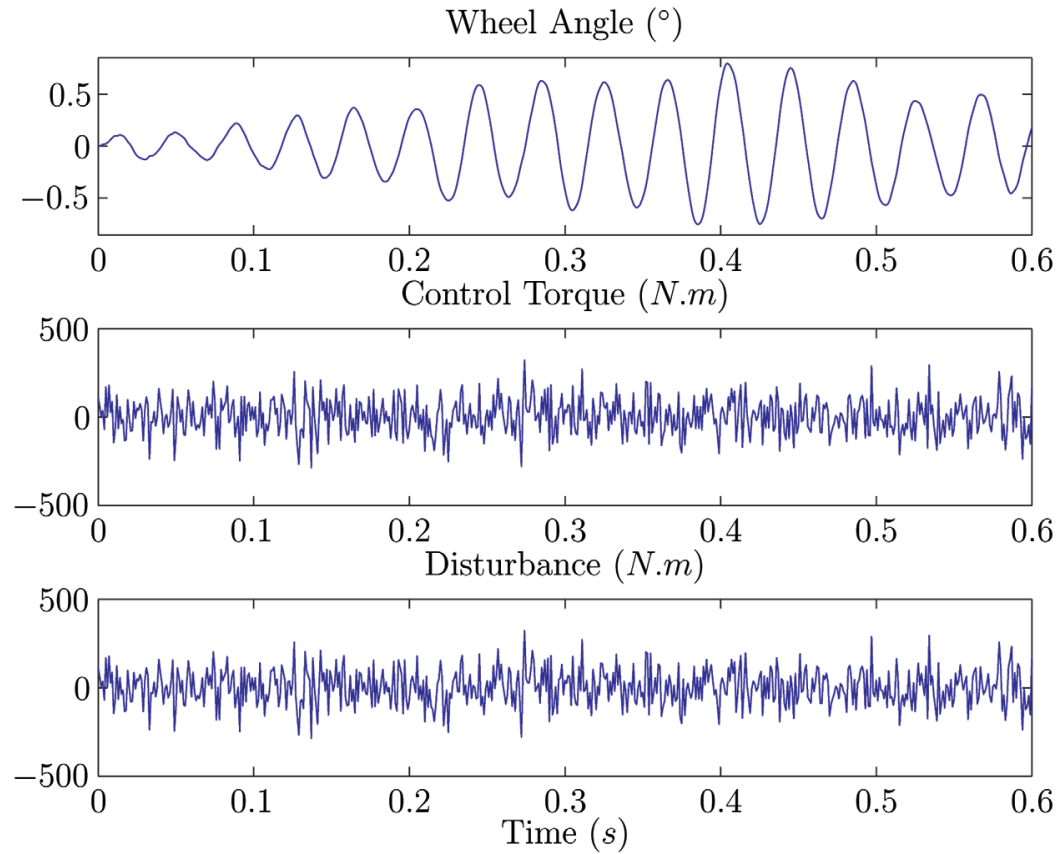

Fig. 13. Active shimmy damping Scenario 2 (PID solution).
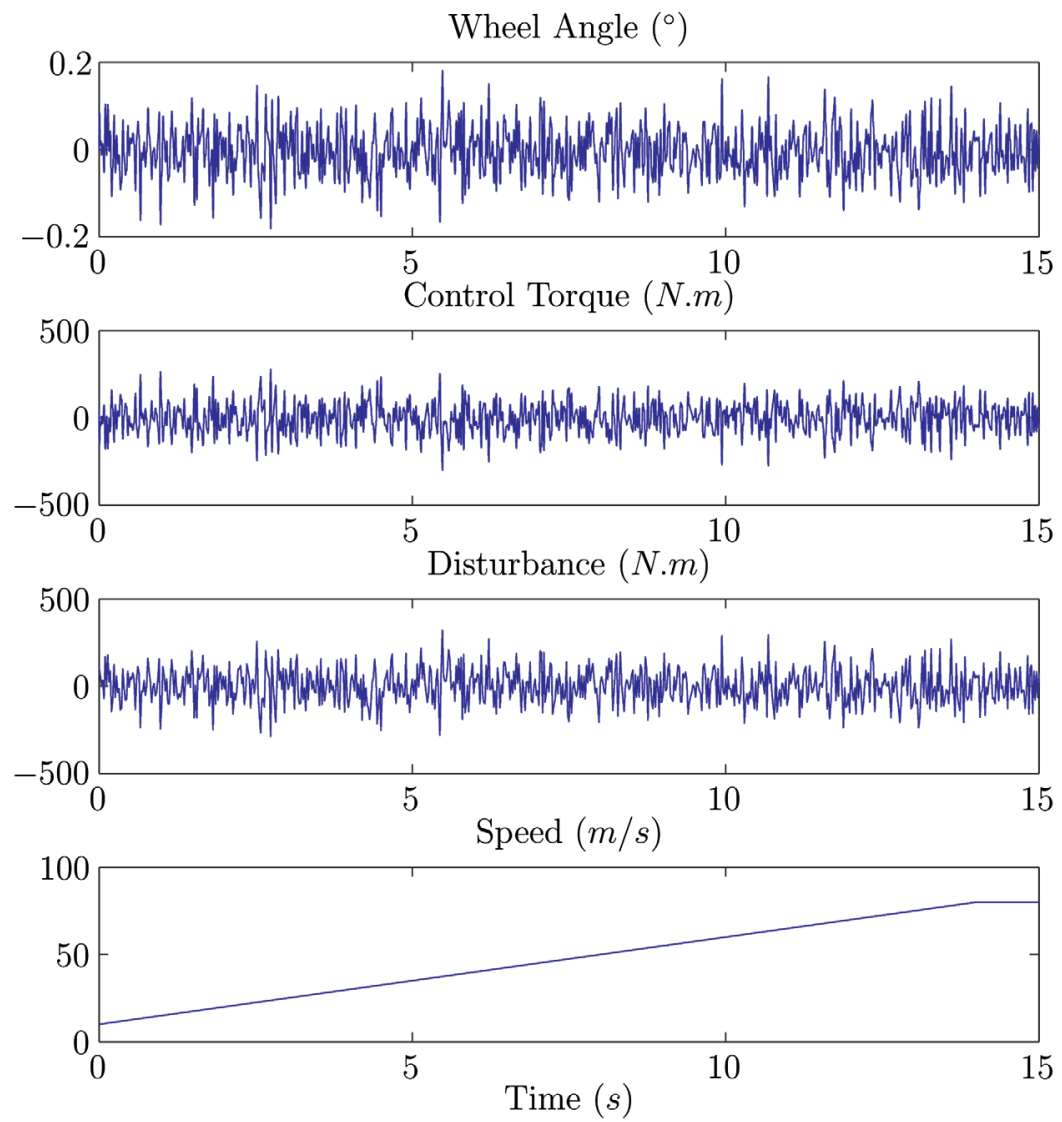

Fig. 14. Active shimmy damping Scenario 3 (Indirect solution). 

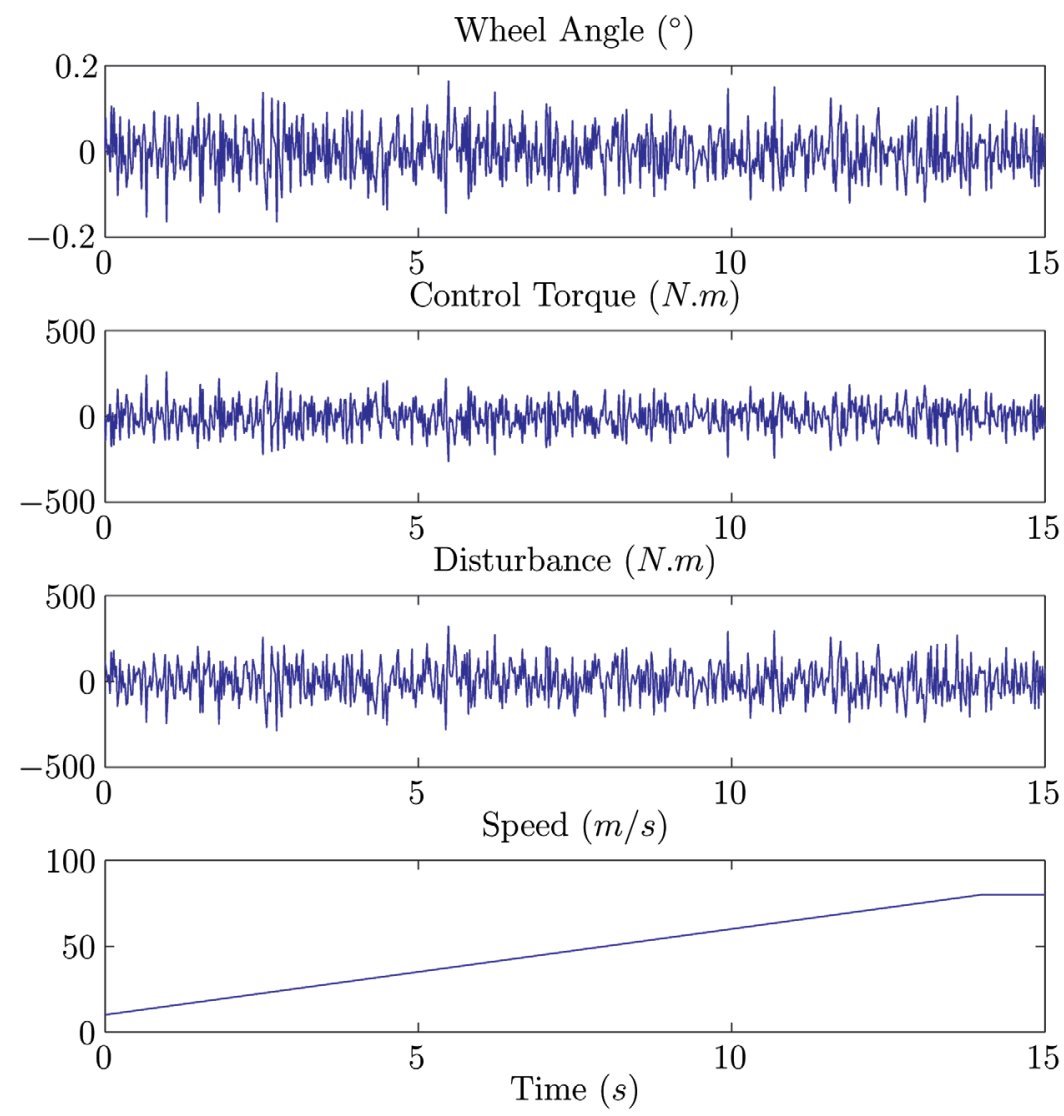

Fig. 15. Active shimmy damping Scenario 3 (Direct solution).

to damp the oscillations ( $15 \mathrm{~s}$ are needed to come over the shimmy oscillations). Thirdly, the periodical control torque required in this case can have strong effects on the actuator.

\subsection{Robustness Analysis of the Adaptive Control Solutions}

The two proposed fuzzy adaptive control solutions show satisfactory results but the variations of the system parameters are not taken into account during simulation tests. Indeed, the conditions at the wheel/road interface are not always the same; the runway can be dry or wet. In addition, some components of the NLG varies during the life of the system. Finally, these variations must be taken into account. That is why a robustness study is proposed. This robustness study, which is in this paper based on the indirect state feedback algorithm, aims at testing the properties of the closed loop system when variations of the parameters are introduced during simulations. Similar results have been obtained with the direct state feedback algorithm.

In the previous section, simulations which correspond to different perturbations that can destabilize the system have been proposed (a pulse disturbance acting during $0.1 \mathrm{~s}$ and a random noise of low amplitude) and the effectiveness of the control loops have been highlighted. However, the tuning of the controllers has been made by considering a fixed value of the parameters of the landing gear. Here, different tests are run in order to analyze the performances and the stability of the algorithms when variations of model parameters are considered.

The proposed study will take an interest to 4 different aspects affecting the system:

- the tire,

- the load,

- the speed,

- the NLG stiffness.

The tire has different behaviors depending on the tire/road interface. The generated forces and moments change if the runway is dry, wet or icy [19]. Thus, three different forces and moments generated at the wheel/road interface are included in the study of robustness. These three features called $F_{y}^{1} / M_{z}^{1}, F_{y}^{2} / M_{z}^{2}$ and $F_{y}^{3} / M_{z}^{3}$ correspond to a dry, wet or snowy runway.

As mentioned earlier, the load $F_{z}$ was fixed. But this value is not constant during the landing and take off and 

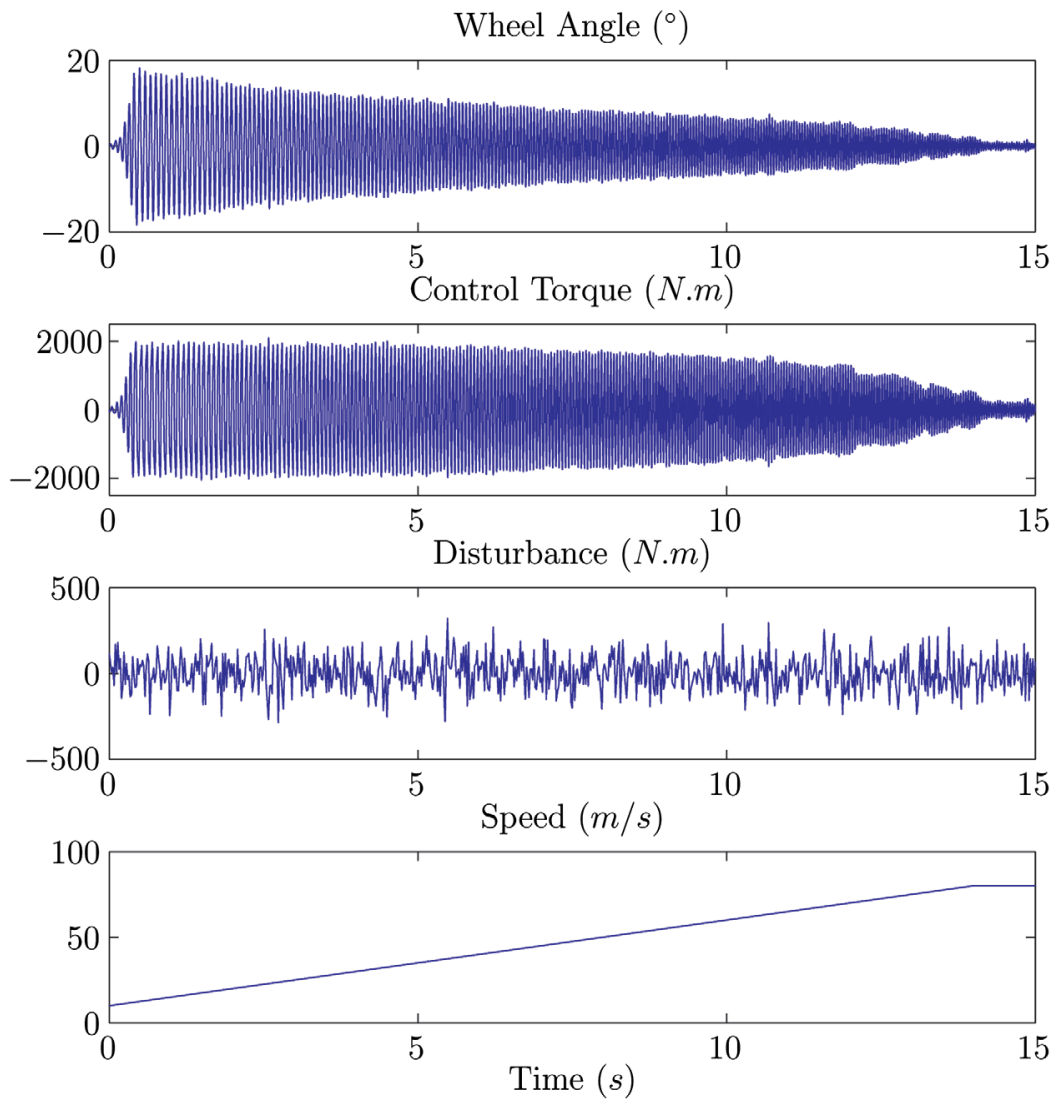

Fig. 16. Active shimmy damping Scenario 3 (PID solution).

can vary depending on the load transfer. The robustness study focuses on a variation of $\pm 20 \%$ of the nominal load. Thus, $F_{z}^{\text {inf }}$ et $F_{z}^{\text {sup }}$ are the two types of considered loads.

The A/C longitudinal speed plays an important role in the outbreak of the shimmy phenomenon. This phenomenon appears beyond $25 \mathrm{~m} / \mathrm{s}$ and increases with respect to the speed. Thus, simulations are performed considering 3 different speeds $v^{1}=20 \mathrm{~m} / \mathrm{s}, v^{2}=50 \mathrm{~m} / \mathrm{s}$ and $v^{3}=80 \mathrm{~m} / \mathrm{s}$.

The mechanical characteristics of the NLG are in reality not constant and the parameters such as the stiffness vary. That is why the study of robustness considers a variation of $\pm 10 \%$ of this constant defining $k^{\text {inf }}$ and $k^{\text {sup }}$.

The combination of all parameter variations has been studied. Thus, a total of 36 simulations has been conducted to validate the robustness of the algorithms. Table 1 that presents these simulation cases is detailed in Section 8 .

The validation of the control solution is based on 4 criteria:

- the maximum wheel angle caused by the perturbation should not exceed $1.5^{\circ}$,
- the effect of the disturbance should not be in evidence after $0.2 s$,

- the torque required to damp the oscillations should not exceed $2000 \mathrm{Nm}$,

- the value of the damping factor $\zeta$.

The damping factor $\zeta$ is calculated by considering the amplitude of two successive oscillations (cf (55)).

Simulation results show that criteria 1,2 and 3 are easily satisfied. When the landing gear parameters vary considering the different cases, the control signal is able to eliminate the shimmy oscillations which appear after the disturbance. Criterion 4 which corresponds to the damping factor is a numerical criterion, evaluating the damping performances of the controller. It allows to compare the results for different simulations. To simplify the results presentation, only the plots of the best/worst cases are presented in this section. Case 32, presented in Fig. 17 is the most damped closed-loop system while case 11, presented in Fig. 18, corresponds to the less damped closed-loop system.

The study of the damping factor shows that the stiffness of the NLG influences the behavior of the 

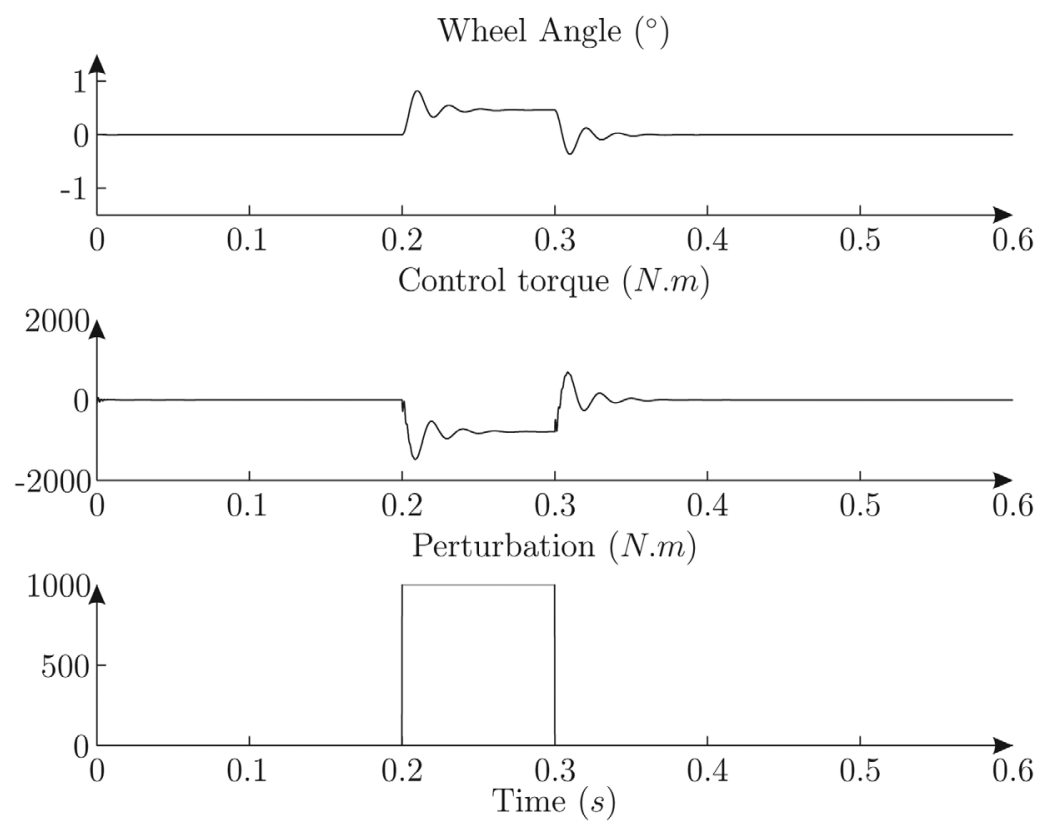

Fig. 17. Case 32 : System which is the more damped $(\zeta=0.209)$.
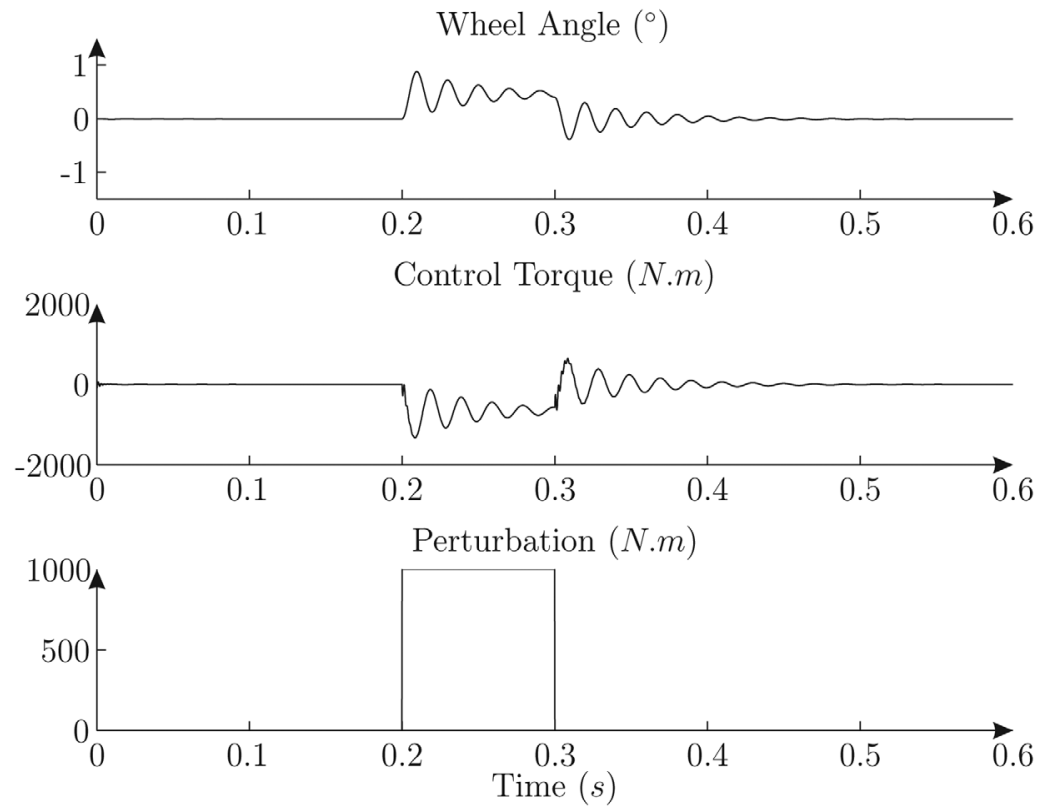

Fig. 18. Case 11 : System which is the less damped $(\zeta=0.07)$.

control solution. Indeed, it can be logically noticed that the simulations using the stiffness equal to $k^{\text {inf }}$ have more oscillations than the simulations using $k^{\text {sup }}$. In addition, the aircraft speed is also an important factor. An increase of $v$ leads to a deterioration of the performances. Simulations done at $20 \mathrm{~m} / \mathrm{s}$ help to obtain an important damping while it is lower at $80 \mathrm{~m} / \mathrm{s}$.

\subsection{Discussion}

\subsubsection{General Properties of Indirect and Direct Controllers}

This section compares the proposed shimmy damping control algorithms according to different criteria (structure, performance, tuning difficulty, ...). The structure of 
fuzzy state feedback adaptive controllers is more complex than traditional PID loops, referring to the adaptive control schemes described in Fig. 6 and Fig. 7. For the indirect adaptive solution, a fuzzy identifier mechanism produces a model of the plant. Then, this model is used to adapt the controller. For the direct adaptive solution, the model of the plant is not necessary, since the controller parameters are directly adapted. Each solution has a specific structure, however, similar components are used in both: a fuzzy identifier, an update law, a feedback linearization control term and a sliding mode control term. The indirect and direct adaptive solutions require restrictive assumptions (measurement of all the states, bounded errors, ...) and the difference of structure leads to specific assumptions for each solution. First, the direct and indirect solutions differ in the number of fuzzy identifiers. The direct solution requires only one fuzzy system which allows the identification of the unknown control function. By contrast, the indirect solution generally requires two fuzzy systems which approximate the two functions $a(\mathbf{x})$ and $b(\mathbf{x})$, representative of the unknown nonlinear dynamics of the system. This difference is generally significant in terms of time computation. Secondly, due to the certainty equivalence control term of (19), the singularity problem must not appear in the indirect solution. Thus, the fuzzy system must ensure that $\hat{b}(\mathbf{x})$ is bounded away from zero. Thirdly, the direct adaptive solution requires a bounding condition for the derivative of nonlinear and smooth functions $b(\mathbf{x})$ of (13).

\subsubsection{Specificities of the Proposed Controllers}

Nevertheless, the specificities of the system to be controlled lead to particular structures of the indirect and direct solutions. The model of the NLG is specific insofar as the term $b(\mathbf{x})$ in (13) is independent of the system's states such that $b(\mathbf{x})=B$. As a consequence, the structure of the algorithms developed in this paper has been simplified. Indeed, a number of drawbacks of the two algorithms disappear. First, the bounding condition of $\dot{b}(\mathbf{x})$ has no meaning. Secondly, the two solutions require only one fuzzy system (only $a(\mathbf{x})$ is identified in the indirect solution) and thus, time computation for the indirect solution is reduced. Thirdly, the problem of singularity does not exist, considering that $B$ is a constant defined by the structural non-zero parameters of the system. If, in this paper, this parameter remains constant, one strategy could be to overcome this assumption. [18] suggest to overspecify the fuzzy system design and to consider $B$ as a function of the states $\mathbf{x}$. This choice may allow greater adaptation flexibility in particular cases.

\subsubsection{Results Discussion}

As for performance, the paper illustrates that the two adaptive controllers give satisfying and similar results in various test conditions. For scenario 1 (constant ground speed, pulse disturbance), the perturbation is rejected and the oscillations disappear rapidly; the system will not be damaged. For scenarios 2 and 3, the roughness of the runway does not affect the NLG and no shimmy is observed, even above the critical aircraft forward velocity.

Another aspect of this study is to specify the characteristics (torque required, bandwidth, ...) of the electromechanical actuator used for shimmy damping. In Fig. 8 and Fig. 9, it can be seen that both controllers require the same control torque in terms of amplitude (approximately 1500 N.m). In fact, a slight difference can be noticed in favor of the direct solution to achieve the same level of performance. Finally, Fig. 16 shows that the PID control strategy, simpler than the fuzzy adaptive controllers proposed in this paper, does not provide good results to damp the shimmy phenomenon. Moreover, in some situations (see Fig. 16), shimmy appears before the critical forward velocity, due to the control action of the PID.

\subsubsection{States Measurements}

As it is presented in this paper, the proposed state feedback control solution requires the measure of 5 signals. Theses measures are:

- the rotation angle of the turning tube $\left(\psi_{a}\right)$ : this signal is already measured for NLG steering purposes with RVDT sensors (Rotary Variable Differential Transformer).

- the rotation speed of the turning tube $\left(\dot{\psi}_{a}\right)$ : this signal can be easily obtained from the rotation angle or a dedicated sensor could be added on the NLG.

- the rotation angle of the wheel $\left(\psi_{w}\right)$ : an RVDT sensor can also be used here. Nevertheless, the measure is more difficult to obtain insofar as the sensor will be in a very noisy environment.

- the rotation speed of wheel $\left(\dot{\psi}_{w}\right)$ : a sensor similar to that used for the measure of $\dot{\psi}_{a}$ can be used here considering the same limitations.

- the lateral displacement of the wheel $\left(y_{l}\right)$ : this state is probably the most difficult to obtain. The lateral displacement is directly linked to the tire slip angle and today many studies devoted to the accurate measurement or estimation of the slip angle are available ([15] and [14]).

Thus, currently the only existing measure is the rotation angle of the turning tube. Concerning the rotation 
speed of the turning tube, the rotation angle and velocity of the wheel, it seems that these measures can be obtained without much difficulties. Finally, the lateral displacement of the wheel is difficult to measure that is why a real time estimation could be investigated.

However the integration of many sensors implies a significant cost that is not always accepted by the aircraft manufacturers. Control solutions requiring less measures should be investigated in the future.

\section{Conclusions}

This paper has presented shimmy, an oscillatory phenomenon which can induce severe structural damages to an aircraft landing gear. This paper describes a control oriented extension of a well known nonlinear shimmy model. This extension concerns two main points. On the one hand, an electrical actuator model is added, in order to develop active control solutions. On the other hand, the initial tire model based on a piecewise linear representation of the lateral force is replaced by a nonlinear description based on a sigmoid curve to represent the lateral forces versus the slip angle. In a traditional way, to counteract the oscillatory phenomenon, passive solutions based on the integration of a damper are added on the NLG. Here, to overcome the main drawback of the classical solutions (non adaptive depending on external conditions), two active shimmy damping control strategies have been developed and the simulation results showing their efficiency are presented. These solutions are also compared with a PID solution. The two active solutions, based on indirect and direct fuzzy adaptive state feedback linearization theories help to damp shimmy satisfactorily, and to define important specifications of the electromechanical actuator (mechanical torque, bandwidth, ...). Even if the applicability of feedback linearization is limited due to the necessary detailed knowledge of the system, the particularities of the NLG model (control matrix $b(\mathbf{x})$ is state-independent) allow a number of simplifications in the structure of the two algorithms. The indirect adaptive fuzzy control uses an identifier mechanism producing a model of the plant which is then used to specify the controller. The direct adaptive fuzzy control does not need the modeling of the plant; instead, the controller parameters are directly estimated.

The performances of the indirect and direct fuzzy algorithms are presented with different disturbance forms (pulse disturbance corresponding to a tire damage and random disturbance representing the roughness of the runway) and forward aircraft velocity profiles. The simulation results are satisfactory, taking into consideration the damping results obtained.
Moreover, a control solution based on a PID has been tested and compared with the two adaptive solutions. The different simulations show that the results obtained with this solution are not satisfactory and in some situations the controller destabilizes the system and generates shimmy before the critical speed.

The two adaptive control solutions give satisfying results but the number of parameters may render difficult the use of such algorithms. Moreover, to obtain an accurate tuning of the controller parameters, the manual tuning is certainly not adequate. Then, it could be interesting to opt for an optimal tuning of the fuzzy adaptive parameters. For example, based on genetic algorithms and particular test scenarios which are representative of the use of the system, the controller can be adjusted automatically. Firstly, the optimization of the adaptive law could be considered. Secondly, both the adaptive law and the fuzzy system may be optimized.

Except for the model simplifications, based on the fact that the control gain is independent of the system's states, one critical point concerns the availability of all the states. For the direct as well as the indirect solution, it is assumed that the five states of the NLG (the rotation speed of the turning tube $\left(\dot{\psi}_{a}\right)$, the rotation angle of the turning tube $\left(\psi_{a}\right)$, the rotation speed of wheel $\left(\dot{\psi}_{w}\right)$, the rotation angle of the wheel $\left(\psi_{w}\right)$ and the lateral displacement of the wheel $\left.\left(y_{l}\right)\right)$ are measured. This provides a good knowledge of the tire dynamics and of the rotational dynamics around the vertical axis at the lower and upper parts of the NLG. This is clearly a constraint for the implementation of the proposed control solution, as a number of measurements may be difficult to obtain in practice. However, this study helps to prove that active shimmy damping is possible, taking account of the actuator constraints. It also helps to evaluate the best performances that could be obtained with an increased knowledge of the plant and can be used to validate future developments. In particular, output feedback controllers using only the measurement of the wheel rotational angle will be developed and compared with the state feedback controllers presented here.

The simulation results showed the need for an important control torque with respect to applied disturbances. The electromechanical actuator, providing this torque, could be composed of a high speed and low torque electrical motor coupled with a mechanical transmission system dedicated to lowering speed and increasing torque. The integration of this mechanical transmission implies structural changes in the NLG modifying its behavior according to the shimmy phenomenon. As a consequence, future investigations will concern the improvement of the actuator model taking account of the mechanical part and the evaluation of the proposed control solutions with this new actuator model. 


\section{Acknowledgments}

The research work which led to the results presented in this paper was carried out thanks to the financial support of the European Union through the DRESS project. The authors wish to thank the Messier Bugatti Company and in particular Jean-Pierre Garcia and Stephane Dellac. For more information about the project, please visit the website: http://www.dress-project.eu/.

The authors would also like to thank the reviewers of the European Journal of Control as well as Dr. Salim Labiod from the Jijel University (Algeria) for their constructive remarks allowing to enhance the quality of this article.

\section{References}

1. Besselink IJM. Shimmy of aircraft main landing gears. $\mathrm{PhD}$ thesis, Delft University of Technology, 2000

2. Brewer HK, Skele P. Active shimmy control system. Technical Report prepared by the Lockheed-California Company, AFFDL-TR-75-136, 1975

3. Chen B, Liu X, Tong S. Adaptive fuzzy output tracking control of MIMO nonlinear systems by backstepping approach. IEEE Trans Fuzzy Syst 2007; 15: 287-300

4. Chen B, Tong S, Liu X. Fuzzy approximate disturbance decoupling of MIMO nonlinear systems by backstepping approach. Fuzzy Sets and Systems, 158: 997-1125, 2007

5. Choi SB, Han SS. H $\infty$ control of electrorheological suspension system subjected to parameter uncertainties. Mechatronics 2003; 13: 639-657

6. Ferreira AMD, Barreiros JAL, Barra Jr. W, Brito de Souza JR. A robust adaptive LQG/LTR TCSC controller applied to damp power system oscillations. Electric Power Systems Research, 2006

7. Goodwine B, Stépán G. Controlling unstable rolling phenomena. J Vib Control 2000; 6: 137-158

8. Høgsberg JR, Krenk S. Linear control strategies for damping of flexible structures. J Sound Vib 2006; 293: 59-77

9. Houlston PR, Garvey SD, Popov AA. Modal control of vibration in rotating machines and other generally damped systems. J Sound Vib 2006; 302: 104-116

10. Huynh T-H, Pouly G, Lauffenburger J-P, Basset M. Active shimmy damping using direct adaptive fuzzy control. 17th IFAC World Congress, 2008. 54

11. Kawabe H, Tsukiyama N, Yoshida K. Active vibration damping based on neural network theory. Materials Science and Engineering 2006; 442: 547-550

12. Kosko B. Fuzzy systems as universal approximators. IEEE Trans Comput 1994; 43: 1329-1333

13. Labiod S, Guerra T-M. Adaptive fuzzy control of a class of SISO nonaffine nonlinear systems. Fuzzy Sets \& Systems 2007; 158: 1098-1126
14. Lamy C, Basset M, Romieu P. Estimation of tyre slip angle and wheel rim edge plane motion by integration of inertial and gps measures at the wheel with an extended kalman filter. 15th international Conference and Exhibition on vehicle dynamics (Safety, Driving pleasure, Braking and Comfort), 2010

15. Lamy C, Caroux J, Basset M, Gissinger GL, Poli D, Romieu P. Comparison of optical and gps based tire slip angle estimation advances in automotive control. Fifth IFAC Symposium on Advances in Automotive Control, 2007

16. Li H-X, Tong SC. A hybrid adaptive fuzzy control for a class of nonlinear mimo systems. IEEE Trans Fuzzy Syst 2003; 11: 24-34

17. Lot R. A motorcycle tire model for dynamic simulations: Theoretical and experimental aspects. Meccanica 2004; 39 : 207-220

18. Ordonez R, Zumberge J, Spooner JT, Passino KM. Adaptive fuzzy control: Experiments and comparative analysis. IEEE Trans Fuzzy Syst 1997; 5: 167-188

19. Pacejka HB. Tyre and Vehicle Dynamics. Butterworth Heinemann, 2006

20. Porcel A, Laurence P, Basset M, Gissinger GL. Tyre model for vehicle simulation: overview and real time solution for critical situations. Proceedings of the 2001 IEEE International Conference on Control Applications, 2001. (CCA '01), pages 817-822, 2001

21. Pouly G, Huynh T-H, Lauffenburger J-P, Basset M. Indirect fuzzy adaptive control for active shimmy damping. 17th IFAC World Congress, 2008

22. Somieski G. Shimmy analysis of a simple aircraft nose landing gear model using different mathematical methods. Aerosp Sci Technol 1997; 87: 545-555

23. Spooner JT, Passino KM. Stable adaptive control using fuzzy systems and neural networks. IEEE Trans Fuzzy Syst 1996; 4: 339-359

24. Stépán G. Chaotic motion of wheel. Veh Syst Dyn 1991; 20 : 341-351

25. Strogatz SH. Nonlinear Dynamics and Chaos: With Applications in Physics, Biology, Chemistry, and Engineering. Addison Wesley Publishing, 1994

26. Sura NK, Suryanarayan S. Stability and response studies on simplified models of nose-wheel landing gear with hard tires. J Aerosp Eng 2004; 85: 29-36

27. Yang YS, Feng G, Ren JS. A combined backstepping and small-gain approach to robust adaptive fuzzy control for strict-feedback nonlinear systems. IEEE Trans Syst Man Cybern A, Syst Humans 2004; 34: 406-420

28. Zefran M, Burdick JW. Stabilization of systems with changing dynamics by means of switching. Proceedings of IEEE Trans Robot Autom 1998; 2: 1090-1095

29. Zhou Y-S, Lai L-Y, Yuan C, Li C. Optimal design for fuzzy controllers by genetic algorithm. IEEE Trans Ind Appl 2000 


\section{Appendix}

Table 1. Combination of the parameter variations

\begin{tabular}{|c|c|c|c|c|}
\hline Case & Runway state & Load & speed & Stiffness \\
\hline 1 & $F_{y}^{1} / M_{z}^{1}$ & $F_{z}^{i n f}$ & $v^{1}$ & $k^{i n f}$ \\
\hline 2 & $F_{y}^{1} / M_{z}^{1}$ & $F_{z}^{i n f}$ & $v^{1}$ & $k^{\text {sup }}$ \\
\hline 3 & $F_{y}^{1} / M_{z}^{1}$ & $F_{z}^{i n f}$ & $v^{2}$ & $k^{i n f}$ \\
\hline 4 & $F_{y}^{1} / M_{z}^{1}$ & $F_{z}^{i n f}$ & $v^{2}$ & $k^{\text {sup }}$ \\
\hline 5 & $F_{y}^{1} / M_{z}^{1}$ & $F_{z}^{i n f}$ & $v^{3}$ & $k^{i n f}$ \\
\hline 6 & $F_{y}^{3} / M_{z}^{3}$ & $F_{z}^{i n f}$ & $v^{3}$ & $k^{\text {sup }}$ \\
\hline 7 & $F_{y}^{1} / M_{z}^{1}$ & $F_{z}^{\text {sup }}$ & $v^{1}$ & $k^{\text {inf }}$ \\
\hline 8 & $F_{y}^{1} / M_{z}^{1}$ & $F_{z}^{\text {sup }}$ & $v^{1}$ & $k^{\text {sup }}$ \\
\hline 9 & $F_{y}^{1} / M_{z}^{1}$ & $F_{z}^{s u p}$ & $v^{2}$ & $k^{i n f}$ \\
\hline 10 & $F_{y}^{1} / M_{z}^{1}$ & $F_{z}^{s u p}$ & $v^{2}$ & $k^{\text {sup }}$ \\
\hline 11 & $F_{y}^{1} / M_{z}^{1}$ & $F_{z}^{\text {sup }}$ & $v^{3}$ & $k^{\text {inf }}$ \\
\hline 12 & $F_{y}^{1} / M_{z}^{1}$ & $F_{z}^{\text {sup }}$ & $v^{3}$ & $k^{s u p}$ \\
\hline 13 & $F_{y}^{2} / M_{z}^{2}$ & $F_{z}^{i n f}$ & $v^{1}$ & $k^{i n f}$ \\
\hline 14 & $F_{y}^{2} / M_{z}^{2}$ & $F_{z}^{i n f}$ & $v^{1}$ & $k^{s u p}$ \\
\hline 15 & $F_{y}^{2} / M_{z}^{2}$ & $F_{z}^{i n f}$ & $v^{2}$ & $k^{i n f}$ \\
\hline 16 & $F_{y}^{2} / M_{z}^{2}$ & $F_{z}^{i n f}$ & $v^{2}$ & $k^{\text {sup }}$ \\
\hline 17 & $F_{y}^{2} / M_{z}^{2}$ & $F_{z}^{i n f}$ & $v^{3}$ & $k^{i n f}$ \\
\hline 18 & $F_{y}^{2} / M_{z}^{2}$ & $F_{z}^{i n f}$ & $v^{3}$ & $k^{\text {sup }}$ \\
\hline 19 & $F_{y}^{2} / M_{z}^{2}$ & $F_{z}^{s u p}$ & $v^{1}$ & $k^{i n f}$ \\
\hline 20 & $F_{y}^{2} / M_{z}^{2}$ & $F_{z}^{\text {sup }}$ & $v^{1}$ & $k^{\text {sup }}$ \\
\hline 21 & $F_{y}^{2} / M_{z}^{2}$ & $F_{z}^{\text {sup }}$ & $v^{2}$ & $k^{\text {inf }}$ \\
\hline 22 & $F_{y}^{2} / M_{z}^{2}$ & $F_{z}^{\text {sup }}$ & $v^{2}$ & $k^{\text {sup }}$ \\
\hline 23 & $F_{y}^{2} / M_{z}^{2}$ & $F_{z}^{\text {sup }}$ & $v^{3}$ & $k^{i n f}$ \\
\hline 24 & $F_{y}^{2} / M_{z}^{2}$ & $F_{z}^{\text {sup }}$ & $v^{3}$ & $k^{\text {sup }}$ \\
\hline 25 & $F_{y}^{3} / M_{z}^{3}$ & $F_{z}^{i n f}$ & $v^{1}$ & $k^{\text {inf }}$ \\
\hline 26 & $F_{y}^{3} / M_{z}^{3}$ & $F_{z}^{i n f}$ & $v^{1}$ & $k^{\text {sup }}$ \\
\hline 27 & $F_{y}^{3} / M_{z}^{3}$ & $F_{z}^{i n f}$ & $v^{2}$ & $k^{\text {inf }}$ \\
\hline 28 & $F_{y}^{3} / M_{z}^{3}$ & $F_{z}^{i n f}$ & $v^{2}$ & $k^{s u p}$ \\
\hline 29 & $F_{y}^{3} / M_{z}^{3}$ & $F_{z}^{i n f}$ & $v^{3}$ & $k^{i n f}$ \\
\hline 30 & $F_{y}^{3} / M_{z}^{3}$ & $F_{z}^{i n f}$ & $v^{3}$ & $k^{\text {sup }}$ \\
\hline 31 & $F_{y}^{3} / M_{z}^{3}$ & $F_{z}^{\text {sup }}$ & $v^{1}$ & $k^{\text {inf }}$ \\
\hline 32 & $F_{y}^{3} / M_{z}^{3}$ & $F_{z}^{\text {sup }}$ & $v^{1}$ & $k^{\text {sup }}$ \\
\hline 33 & $F_{y}^{3} / M_{z}^{3}$ & $F_{z}^{\text {sup }}$ & $v^{2}$ & $k^{i n f}$ \\
\hline 34 & $F_{y}^{3} / M_{z}^{3}$ & $F_{z}^{\text {sup }}$ & $v^{2}$ & $k^{\text {sup }}$ \\
\hline 35 & $F_{y}^{3} / M_{z}^{3}$ & $F_{z}^{\text {sup }}$ & $v^{3}$ & $k^{i n f}$ \\
\hline 36 & $F_{y}^{3} / M_{z}^{3}$ & $F_{z}^{s u p}$ & $v^{3}$ & $k^{s u p}$ \\
\hline
\end{tabular}

